\title{
PHYSICAL AND MATHEMATICAL DESCRIPTION OF NUCLEAR WEAPONS IDENTIFICATION SYSTEM (NWIS) SIGNATURES
}

J. T. Mihalczo

T. E. Valentine

J. A. Mullens

J. K. Mattingly

RECEIVED

NOV 301998

OSTI

Nuclear Materials Managcment and

Storage Program Office

September 26, 1997

Prepared by the

Oak Ridge X-12 Plant

Oak Ridge, Tennessec 37831 managed by

Lockheed Martin Energy Systems, Inc. for the

U.S. DEPARTMENT OF ENERGY

under contract DE-ACOS-84OR21400 


\section{DISCLAMMER}

This report was prepared as an account of work sponsored by an agency of the United States government. Neither the United States Government nor any agency thereof, nor any of their employees, makes any warranty, express or implied, or assumes any legal liability or responsibility for the accuracy, completeness, or usefulness of any information, apparatus, product, or process disclosed, or represents that its use would not infringe privately owned rights. Reference berein to any specific commercial product, process, or service by trade name, trademark, madufacturer, or otherwise, does not necessarily constitute or imply its endorsement, recommendation, or favoring by the United States Governmeat or any agency thereof. The views and opinions of authors expressed herein do not necessarily state or reflect those of the United States Government or any agency thereof.

\section{COPYRIGHT NOTICE}

The submitted manuscript bas been anthored by a contractor of the U.S. Government under contract DE-ACOS-84OR21400. Accordingly, the U.S. Government retains a paid-up, noverclusive, irrevocable, worldwide license to publish or reproduce the pablished form of this contribution, prepare derivative works, distribute copies to the public, and perform publicly and display publicly, or allow others to do so, for U.S. Government purpose 


\section{DISCLAIMER}

Portions of this document may be illegible in electronic image products. Images are produced from the best available original document. 


\title{
PHYSICAL AND MATHEMATICAL DESCRIPTION OF NUCLEAR WEAPONS IDENTIFICATION SYSTEM (NWIS) SIGNATURES
}

\author{
J. T. Mihalczo \\ T. E. Valentine \\ J. A. Mullens \\ J. K. Mattingly
}

\begin{abstract}
Oak Ridge National Laboratory*
P.O. Box 2008
\end{abstract}

Oak Ridge, Tennessee 37831-6004

(423) $574-5577$

September 26, 1997

\footnotetext{
"Managed by Lockheed Martin Energy Research Corporation for the U.S. Department of Energy under contract DE-AC05-96OR22464.
} 



\section{CONTENTS}

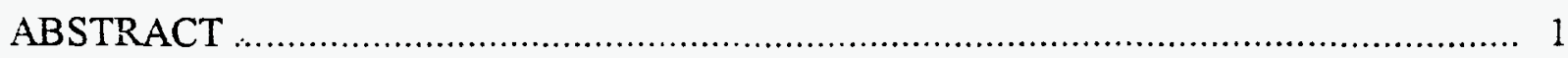

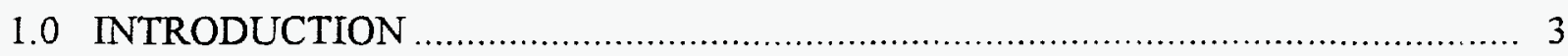

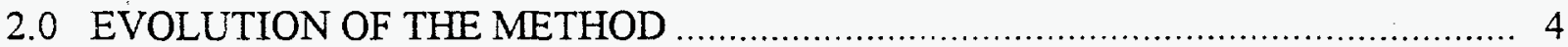

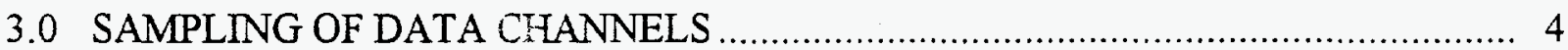

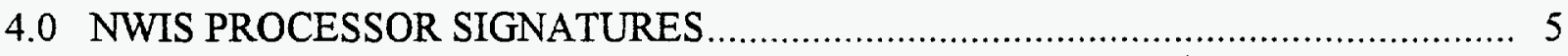

5.0 AUTO CORRELATION FUNCTION OF SOURCE CHANNEL (1) ......................... 6

6.0 AUTO CORRELATION FUNCTION OF DETECTOR CHANNELS (2 \& 3)............. 7

7.0 CROSS CORRELATION FUNCTIONS BETWEEN DETECTOR AND SOURCE..... 8

8.0 CROSS CORRELATION FUNCTIONS BETWEEN DETECTORS .......................... 9

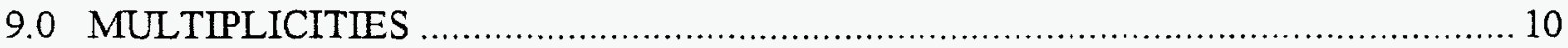

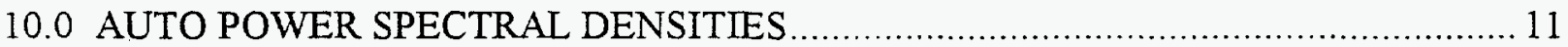

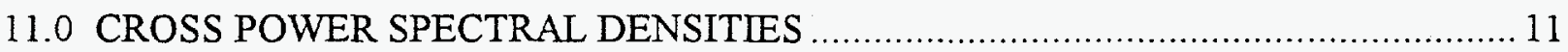

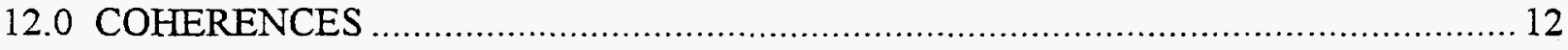

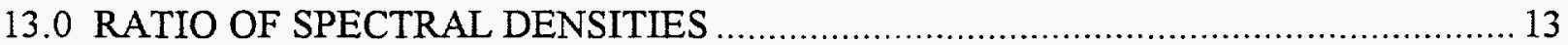

14.0 FREQUENCY ANALYSIS SIGNATURES WITHOUT TRANSMITTED

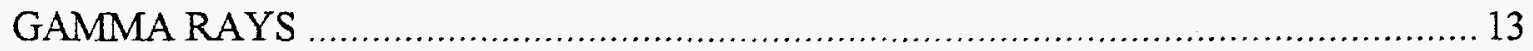

15.0 MONTE CARLO CALCULATION OF SIGNATURES ……................................... 14

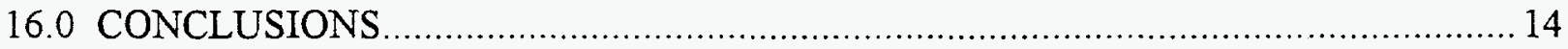

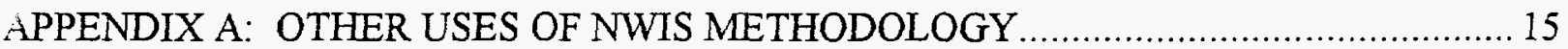

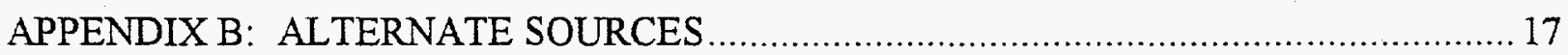

APPENDIX C: AUTO CORRELATION FUNCTION PROCESSING ……....................... 19

APPENDIX D: CROSS CORRELATION FUNCTION PROCESSING .............................. 21

APPENDIX E: NON INTRUSIVE USE FOR IDENTIFICATION OF

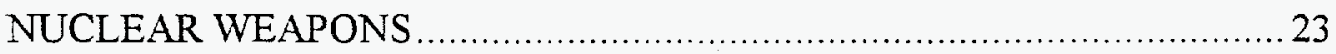

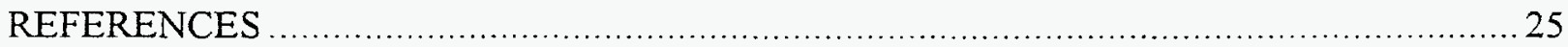




\section{LIST OF FIGURES}

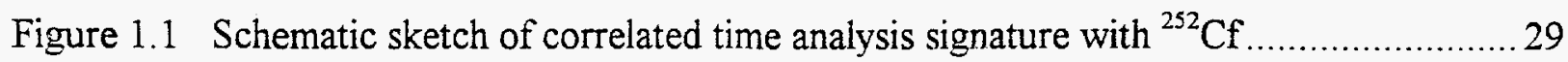

Figure 1.2 Photograph of a portable version of the NWIS processor ....................................30

Figure 1.3 A. Photograph of uranium metal annular casting in a steel tray ............................31

Figure 1.3 B. Photograph of an annular casting in its plastic bag in an unsealed can .............. 31

Figure 1.4 Source detector (4) casting configuration for measurements without moderator....32

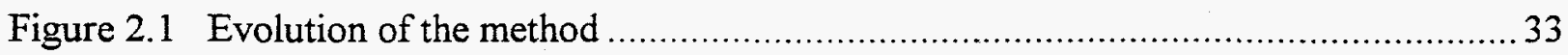

Figure 5.1 Auto correlation function of the $252 \mathrm{Cf}$ source ionization chamber

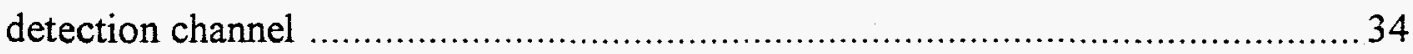

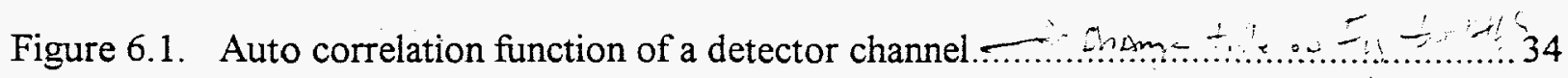

Figure 7.1. Right half $(\tau \geq 0)$ of the cross correlation function between detector

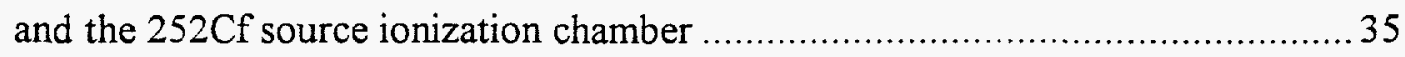

Figure 7.2. Approximation to $\Sigma \times$ : Logarithm of ratio of cross-correlation between source and detector with casting present to source-detector cross-correlation in air

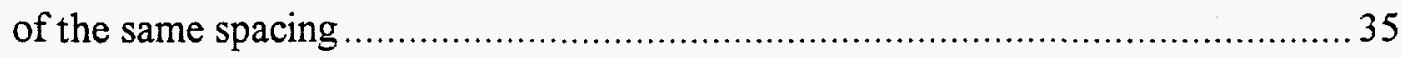

Figure 8.1. Cross correlation function between two detection channels ................................36

Figure 9.1. Multiplicities for the source ionization chamber channel...............................36

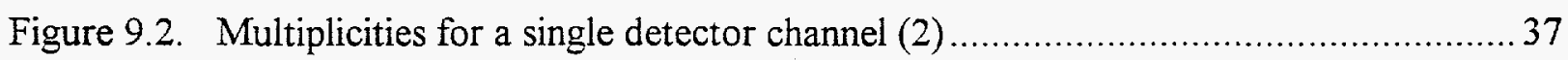

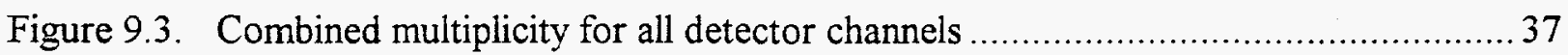

Figure 9.4. Ratios of higher order/singles as a function of doubles to singles ratio for a

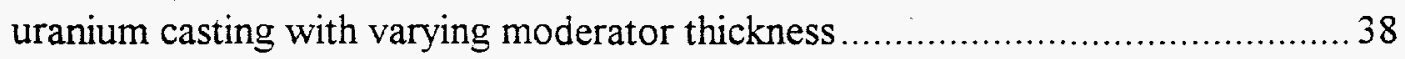

Figure 10.1. APSD $G_{11}$ of the source detection channel as a function of frequency..................38

Figure 10.2. APSD $\mathrm{G}_{22}$ of one of the detector channels as a function of frequency ...................39

Figure 11.1. Cross power spectral density between detector channel 2 and the source

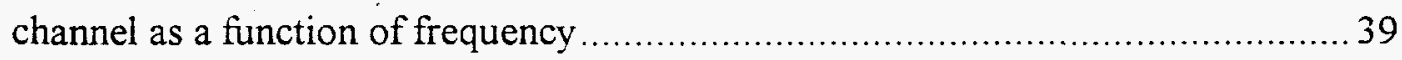

Figure 11.2. Cross power spectral density between detectors as a function of frequency ......... 40

Figure 11.3. Polar plot of CPSD12 imaginary part vs real part ....................................... 40

Figure 12.1. Coherence between a detection channel and the ${ }^{252} \mathrm{Cf}$ source

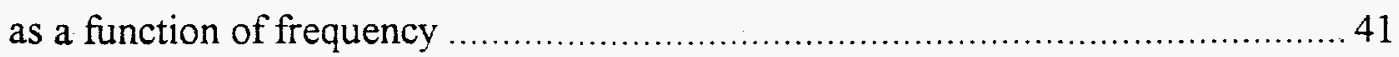

Figure 12.2. Coherence between the two detection channels as a function of frequency .......... 41

Figure 13.1. Ratio of spectral densities as a function of frequency ...................................... 42 


\section{LIST OF FIGURES (continued)}

Figure E.1. NWIS signature acquisition with cart portable system at the Y-12 Plant (1996)

Figure E.2. Ratios of CPSDs between source and detector to the reference set for a weapons component in the set.

Figure E.3. Ratio of CPSD between source and detector to the reference set for a weapons component not in the set. 


\section{LIST OF TABLES}

Table 9.1. Ratios of combined multiplicities for all detectors for a HEU-metal casting ........... 11

Table B. 1. Advantages and disadvantages of ${ }^{252} \mathrm{Cf}$ and APSTNG source ........................... 17

Table C.1. Computation of summation $\sum_{k=0}^{N-1-\tau} \mathrm{X}_{k} \mathrm{X}_{k+\tau}$ for block of 8 time bins....................... 19

Table D. 1. Computation of summation $\sum_{k=0}^{N-1-\tau} X_{k} Y_{k+\tau}$ for block of 8 time bins .....................2 21 


\title{
PHYSICAL AND MATHEMATICAL DESCRIPTION OF \\ NUCLEAR WEAPONS IENTIFICATION SYSTEM (NWIS) SIGNATURES
}

\author{
J. T. Mihalczo, T. E. Valentine, J. A. Mullens, and J. K. Mattingly
}

\begin{abstract}
This report describes all time and frequency analysis parameters measured with the new Nuclear Weapons Identification System (NWIS) processor with three input channels: (1) the ${ }^{252} \mathrm{Cf}$ source ionization chamber; (2) a detection channel; and (3) a second detection channel for active measurements. An intuitive and physical description of the various functions is given as well as a brief mathematical description and a brief description of how the data are acquired. If the full five channel capability is used, the number of functions increases in number but not in type. The parameters provided by this new NWIS processor can be divided into two general classes: time analysis signatures including multiplicities and frequency analysis signatures. Data from measurements with an $18.75 \mathrm{~kg}$ highly enriched uranium $\left(93.2 \mathrm{wt} \%,{ }^{235} \mathrm{U}\right)$ metal casting for storage are presented to illustrate the various time and frequency analysis parameters.
\end{abstract}




\subsection{INTRODUCTION}

The Nuclear Weapons Identification System (NWIS) was developed primarily for identification of nuclear weapons/components that contain highly enriched uranium (HEU) (Ref. 1). For active measurements (i.e. where the nuclear weapons/components are "stimulated"), it utilizes a ${ }^{252} \mathrm{Cf}$ source in an ionization chamber to define the time of spontaneous fission of ${ }^{252} \mathrm{Cf}$ ( 614000 fission per sec per $\mu \mathrm{g}$ ). The neutron source is usually located adjacent to the weapons/ components/ containers with a pair of detectirs on the opposite side of the weapons/components/ containers. Neutrons from the source enter the component inducing fission that produces additional neutron and gamma rays, some of which are detected. The time behavior of the detected events after ${ }^{252} \mathrm{Cf}$ fission in a plastic scintillator sensitive to neutrons and gamma rays without distinction is sketched in Fig. 1.1. The directly transmitted gamma rays arrive at the detector slightly delayed in time from the ${ }^{252} \mathrm{Cf}$ fission. Because all gamma rays travel with the speed of light, the dispersion of this part of the signal in time gives the combined time resolution for detecting the ${ }^{252} \mathrm{Cf}$ spontaneous fission in the ionization and the gamma ray in the detector. This directly transmitted gamma ray component can be used for active gamma ray imaging much like an $\mathrm{x}$-ray image but using gamma rays from spontaneous fission of ${ }^{252} \mathrm{Cf}$ as the source. After the transmitted gamma rays, the neutrons that travel directly from the source through the material without collision arrive at the detector. These have a time dispersion because of the variation in the energy of neutrons from ${ }^{252} \mathrm{Cf}$ fission. The next particles to arrive are the scattered neutrons, and finally both neutrons and gamma rays from induced fission. This figure is a simplification in that the various groups of neutrons overlap in real systems.

NWIS with a ${ }^{252} \mathrm{Cf}$-source basically an active neutron interrogative method that can be used for $\mathrm{HEU}$ and $\mathrm{Pu}$ systems. However, for systems like Pu with a large inherent neutron source from the spontaneous fission of ${ }^{240} \mathrm{Pu}$, NWIS can be employed for passive measurements without the ${ }^{252} \mathrm{Cf}$ source by using the inherent radiation originating in the $\mathrm{Pu}$. These types of active measurements have been used for 15 weapons systems including measurements: with components, with components in containers, with fully assembled weapon systems, and with mockups. The system has beer used successfully to distinguish $\mathrm{Pu}$ weapons components in blind testing at Los Alamos National _aboratory in both the active and passive modes in 1997 and has been shown to have high sensitivity to fissile mass. This system is also useful for other nondestructive assay applications (Refs. 2 and 3), and a variety of other applications some of which are described in Appendix A.

This report describes all time and frequency analysis parameters measured with the new NWIS processor (Ref. 4) with three input channels: (1) the ${ }^{252} \mathrm{Cf}$ source ionization chamber; (2) a detection channel; and (3) a second detection channel. A photograph of a portable version of the processor is shown in Fig. 1.2. An intuitive and physical description of the various functions is given as well as a brief mathematical description and a brief description of how the data are acquired. If the full five channel capability is used, the number of functions increases in number but not in type. The parameters provided by this new NWIS processor can be divided into two general classes: time analysis signatures inclucing multiplicities and frequency analysis signatures. The evolution of this method of measurement and its analogy to previous measurements are presented. Data from measurements with an $18.75 \mathrm{~kg} \mathrm{HEU}\left(93.2 \mathrm{wt} \%,{ }^{235} \mathrm{U}\right)$ standard annular 
metal casting for storage are presented to illustrate the various time and frequency analysis parameters. In these measurements the casting in a sealed steal can was surrounded on all sides, top, and bottom with 3 -in. thick polyethylene reflector except on the side where the plastic scintillation detectors (sensitive to neutrons and gamma rays without distinction) are located. For some of the multiplicity data presented, the reflector thickness around the casting was varied. Photographs of the actual casting are shown in Fig. 1.3, and a casting in a can without reflector is shown in Fig. 1.4 with the source on one side and four detectors on the opposite side.

\subsection{EVOLUTION OF THE METHOD}

The origins of NWIS lie in the pulsed neutron (Ref. 5) and Rossi- $\alpha$ (Ref. 6) measurement methods with fissile material that were initiated in the mid-early 1940 s. The evolution of the method is depicted in Fig. 2.1. Pulsed neutron measurements were initiated in Oak Ridge in 1956 with D-T pulsed neutron generators and Rossi- $\alpha$ and multiplicity measurements were first performed in 1962 at Oak Ridge (Ref. 7). Experiments with HEU metal systems were being performed at the Oak Ridge Critical Experiments Facility in the 1960s using a Cockkroft Walton $D-T$ neutron generator operating as a repetitively pulsed neutron source with pulse repetition rates up to $1 \mathrm{MHz}$ (Ref. 8). In $1968,{ }^{252} \mathrm{Cf}$ was inserted on one plate of a parallel plate ionization chamber to determine the time of each ${ }^{252} \mathrm{Cf}$ fission (Ref. 9). The motivation for this insertion was to obviate the use of the accelerator to produce pulses of neutrons since the accelerator was very difficult to maintain and operate. By measuring the time distribution of events in a scintillation detector after ${ }^{252} \mathrm{Cf}$ fission, the same physics data was acquired as with the accelerator. After much intercomparison of results of both methods of acquiring pulsed neutron data, the accelerator was shut down and abandoned as a pulsed neutron generator. From this time, the randomly pulsed neutron method with ${ }^{252} \mathrm{Cf}$ was used for pulsed neutron measurements. In the mid $1970 \mathrm{~s}$, the advantages of frequency analysis methods were realized, and the first measurements of this type were performed in 1974 with a mockup of the FFTF reactor and in 1975 with HEU metal cylinders (Ref. 10). However, the frequency analysis capability was limited to low sampling and processing rates, large racks of cumbersome equipment, and thus severely limited the application of this method for $U$ and Pu metals. Sampling rates progressed from up to $5 \mathrm{kHz}$ in 1974 , to up to $200 \mathrm{kHz}$ in 1984 , to up to $32 \mathrm{MHz}$ in 1992 and finally to the present rate of up to $1 \mathrm{GHz}$ in 1996. Waveform digitizers were available before 1996 that could sample at this rate but process at much lower rates. The processing rates increased more slowly and for the sampling rate system described here progressed sequentially from $5 \mathrm{kHz}$ in 1973 , to $200 \mathrm{kHz}$ in 1984 , to $1 \mathrm{MHz}$ in 1990 , and to $500 \mathrm{MHz}$ for the present system. Time analysis hardware slowly developed in this time period, but the main system used in time analysis measurements with ${ }^{252} \mathrm{Cf}$ was a commercially available time-to-pulse-height or amplitude converter (TAC) with a multichannel analyzer (MCA). Such a TAC-MCA combination was utilized worldwide in physics research since about 1950 . The new NWIS processor combines both the time and frequency analysis signatures into one compact processor (Fig. 1.2).

\subsection{SAMPLING OF THE DATA CHANNELS}

NWIS uses a high speed data acquisition processor to collect signals from a ${ }^{252} \mathrm{Cf}$ source and detectors (such as plastic scintillators sensitive to neutrons and gamma rays without distinction) 
after the signals have triggered constant fraction discriminators with appropriate thresholds. The constant fraction discriminator provides a timing mark for each incoming pulse that is independent of the incoming pulse height. Signals from the discriminator are acquired by the processor over a large time interval in $256,512,1024$, etc. time bins ${ }^{\dagger}$ that can be as short as 1 nsec. Signals from the three channels are obtained by leading-edge timing of the pulses from the detector system discriminators to obtain a time ( $<1$ nsec resolution) associated with each pulse from the detection system. The three channeis are synchronously sampled and the signals in a time interval are represented by a 1 if a signal is present and 0 if a signal is not present. Leading-edge timing circuitry at the input to the processor chip defines the presence or absence of a pulse within 1 nsec. These many adjacent time intervals form a block of data (for example of 512 points). From these blocks of data the various signatures are obtained.

\subsection{NWIS PROCESSOR SIGNATURES}

For a three channel input system as described in the introduction, the NWIS processor acquires the following three types of signatures.

\section{Time Dependent Signatures}

a. Three auto correlation functions: $\mathrm{AC}_{11}(\mathrm{t}), \mathrm{AC}_{22}(\mathrm{t}), \mathrm{AC}_{33}(\mathrm{t})$

b. Three cross correlation functions: $\mathrm{CC}_{12}(\mathrm{t}), \mathrm{CC}_{13}(\mathrm{t}), \mathrm{CC}_{23}(\mathrm{t})$

Multiplicity Signatures

c. Multiplicities for all three channeis: $M_{1}, M_{2}, M_{3}$, and the total $M$ for all detector channels simultaneously; multiplicity is defined here as the number of detected events in a time interval.

Frequency Dependent Signatures

d. Three auto power spectral densities (APSDs): $\mathrm{G}_{11}(\omega), \mathrm{G}_{22}(\omega), \mathrm{G}_{33}(\omega)$

e. Three cross spectral densities (CPSDs): $G_{12}(\omega), G_{13}(\omega), G_{23}(\omega)$ which in general have real and imaginary parts.

f. Three coherences $\gamma_{12}{ }^{2}, \gamma_{13}{ }^{2}$, and $\gamma_{23}{ }^{2}$ where $\gamma_{i j}{ }^{2}=\left|G_{i j}\right|^{2} / G_{i i} G_{i j}$ where ||$^{2}$ means complex multiplication

g. One ratio of spectral densities $R_{123}(\omega)=G_{12} * G_{13} / G_{11} G_{23}$ where * means complex conjugation.

This set of twenty signatures (excluding count rates) expands to a set of 52 if the full five channel capability is used. Three of these signatures $\left(A C_{11} ; M_{1}\right.$, and $\left.G_{11}\right)$ describe only the operation of the source detection system and can be used to verify its proper operation. From the frequency dependence of the signatures ( $d, e$, and $f$ ), the breakfrequency $\left(f_{b}\right)$ can be obtained and it is related to a single prompt neutron decay constant $\alpha\left(\alpha=2 \pi f_{b}\right)$ if fundamental mode decay predominates. If not, multiple breakfrequencies may be obtained by least squares fitting of the data. For this fitting, the frequency response of the detector systems and processor must be removed from the frequency dependence of the APSDs, $G_{22}(\omega)$ and $G_{33}(\omega)$ and the CPSDs, $G_{12}(\omega), G_{13}(\omega)$ and

These sequences of $\mathrm{N}$ time bins $(\mathrm{N}=256,512,1024$, etc. $)$ are call data blocks. 
$\mathrm{G}_{23}(\omega)$. The frequency response of the detection system can be measured with only the source and detectors. This is usually done in two ways: (1) with the ${ }^{252} \mathrm{Cf}$ source adjacent to the detectors, or (2) with the source and detectors spaced the same distance and configuration as in the actual measurements but without the fissile item between the source and detectors. The frequency response of the measurement system appear in these frequency spectra as products and can easily be divided out. This may be necessary to extract physics information but not for signature comparisons especially ratios where these effects of the measurement system appear in the numerator and denominator and thus cancel. In a sense, these prompt neutron decay constants are additional signatures which also can be used for identification and are related to the fissile mass, presence of other materials, and configuration.

For passive measurements (i.e., no ${ }^{252} \mathrm{Cf}$ source) only quantities involving channel 2 and 3 are obtained: $\mathrm{AC}_{22}, \mathrm{AC}_{33}, \mathrm{CC}_{23}, \mathrm{M}_{2}, \mathrm{M}_{3}, \mathrm{M}, \mathrm{G}_{22}, \mathrm{G}_{33}, \mathrm{G}_{23}$, and $\gamma^{2}{ }_{23}$. Passive measurements are useful for fissile systems with strong inherent neutron sources.

\subsection{AUTO CORRELATION FUNCTION OF THE SOURCE ${ }^{\ddagger}$ CHANNEL (1)}

The auto correlation function (AC) of the ${ }^{252} \mathrm{Cf}$ source ionization chamber channel is mathematically defined as

$$
A C_{x x}(\tau)=\underset{T \rightarrow \infty}{\operatorname{Lim}} \frac{1}{2 T} \int_{-T}^{T} X(t) X(t+\tau) d t
$$

where $X(t)$ is the signal from the source ionization chamber electronics at time $t, X(t+\tau)$ is the signal at time $t+\tau$, and $\tau$ is defined as the lag time. In general, the auto correlation function is a measure of the correlated information in a signal at two different times. In practice, the auto correlation function is estimated from time averages of finite length and is estimated from discrete time signatures. A discrete form of the auto correlation (Eq. 5.1) for a data block of $\mathrm{N}$ points is given by

$$
A C_{x x}(\tau)=\frac{1}{N} \sum_{k=o}^{N-1} X_{k} X_{k+\tau}
$$

where $X_{k}$ is a discrete sample of $X(t)$ at time $t_{k}$ and $X_{k+\tau}$ is a discrete sample of $X(t)$ at time $t_{k}+\tau$.

Because the discretized representation of the correlation function is defined for an finite length of time or finite block of data of $\mathrm{N}$ time bins, the auto correlation will be biased. The unbiased linear auto correlation function is given by

\footnotetext{
${ }^{\ddagger}$ Alternate sources other than ${ }^{252} \mathrm{Cf}$ are discussed in Appendix B.
} 


$$
A C_{x x}^{U L}(\tau)=\frac{1}{N-\tau} \sum_{k=0}^{N-1-\tau} X_{k} X_{k+\tau}
$$

The expected value of the unbiased auto correlation function (Eq. 5.3) is the same as the expected value of the continuous auto correlation function (Eq. 5.1). For a data block of 512 points, there are 512 values of $\tau$ for positive times. The auto correlation functions are the same for both positive and negative values of $\tau$. The NWIS processor acquires the unbiased linear correlation function for time analysis signatures. Of course, this is done over many blocks of data until the desired statistical precision is obtained. The unbiased linear auto correlation functions are used since they also relate to previously measured time domain measurements, such as the Rossi- $\alpha$ (Ref. 6) with a single detector.

Consider the $\mathrm{AC}_{11}(\tau)$ of the source ionization chamber channel. A plot of the unbiased linear auto correlation function, $\mathrm{AC}_{11}$ is given in Fig. 5.1 for a ${ }^{252} \mathrm{Cf}$ source ionization chamber with a ${ }^{252} \mathrm{Cf}$ fission rate of 525000 fissions $/ \mathrm{sec}$. There are various features on this plot designated by letters. The value of the auto correlation function at time $0, \mathrm{AC}_{11}(0)$ [designated by $(\mathrm{a})$ ] is the count rate per ${ }^{252} \mathrm{Cf}$ fission which is normalized to 1 . The actual count rate for the ${ }^{252} \mathrm{Cf}$ source is the sum of all ${ }^{252} \mathrm{Cf}$ fissions recorded during the measurement divided by the time the processor is sampling data which is the number of blocks multiplied by the time period for each block. This average count rate is also printed in a summary table.

Another feature of this signature [designated by (b)] gives the dead time of the ${ }^{252} \mathrm{Cf}$ chamber electronics. This particular feature allows the dead time to be measured at low count rates (Ref. 11). It can also be used at various count rates to determine if the dead time is a function of count rate. These dead times can be obtained without knowing the actual count rate and therefore make dead time measurements much simpler since the true count rate is not needed for this deadtime determination.

Another feature of this signature [designated by (c)] is the constant background value for positive times. The amplitude of the constant part should be the detected fission rate of the ${ }^{252} \mathrm{Cf}$ source squared multiplied by the sampling interval. Since this auto correlation function is expressed in units of per ${ }^{252} \mathrm{Cf}$ fission, this amplitude equals the detected ${ }^{252} \mathrm{Cf}$ fission rate of the source multiplied by the sampling interval. This function can also be used to evaluate the source detection channel performance. If there is multiple pulsing, there will be sharp peaks at the appropriate times in the constant part of the signal for positive lag times immediately after the recovery from the dead time or thereafter depending on their time of occurrence.

\subsection{AUTOCORRELATION FUNCTION OF DETECTOR CHANNELS}

The auto correlation function of a detector channel is mathematically defined analogously to Eq. 5.1 but with $\mathrm{X}(\mathrm{t})$, the signal from the ${ }^{252} \mathrm{Cf}$ source ionization channel, replaced by $\mathrm{Y}(\mathrm{t})$ or $\mathrm{Z}(\mathrm{t})$ the signals from each of the detectors. Thus for a detection channel 


$$
A C_{y y}(\tau)=\operatorname{Lim}_{T \rightarrow \infty} \frac{1}{2 T} \int_{-T}^{T} Y(t) Y(t+\tau) d t
$$

where $\mathrm{Y}(\mathrm{t})$ is the signal from detection system 2 at time $(\mathrm{t})$. For discrete data points, this integral is replaced by a summation analogous to those Eqs. 5.3. The auto correlation function of the other detector channel is $\mathrm{AC}_{\mathrm{zz}}(\mathrm{t})$ and is defined by an equation like Eq. 6.1 but with $\mathrm{Y}(\mathrm{t})$ replaced with $Z(t)$.

A typical plot of the unbiased auto linear auto correlation function $\mathrm{AC}_{33}(\tau)$ is given in Fig. 6.1. Some of the features of this function are similar to $\mathrm{AC}_{11}(\tau) . \mathrm{AC}_{33}(0)$ is the count rate per detected ${ }^{252} \mathrm{Cf}$ fission [designated by (a)]. The dead time for the detection system electronics is again designated by (b). The background level at large time lags (d) is the count rate squared times the sampling interval divided by the detected ${ }^{252} \mathrm{Cf}$ fission rate. This normalization is utilized so that the signatures are independent of source fission rate. Except for the normalization of dividing by the ${ }^{252} \mathrm{Cf}$ fission rate, this background is the same as that for a single detector Rossi- $\alpha$ measurement with a Type-I time analyzer (one that does not miss any detector pulses, Ref. 12). This function exhibits additional features over $\mathrm{AC}_{11}(\tau)$ in that it is the equivalent of a single detector Rossi- $\alpha$ measurement and contains the prompt neutron decay [designated by feature (c)] which may be used to obtain the prompt neutron decay constant. The data can be fitted to $\sum_{n} A_{n} \exp \left[-\alpha_{n} t\right]$ to obtain the decay constants $\alpha_{n}$ with or without removing the background (d) which is known. For simple systems where fundamental mode predominates, there will only be one term in the expansion and $\alpha_{0}$ can be obtained. For the data presented in Fig. 6.1, the time decay essentially contains two characteristic decays: a fast decay associated with the casting, and a slower decay that persists beyond $100 \mathrm{nsec}$ associated with the casting surrounded by moderator. An example of the data processing for the single detector Rossi- $\alpha$ measurement compared to that for the auto correlation function is given in Appendix $\mathrm{C}$ for a single block of data.

\subsection{CROSS CORRELATION FUNCTION BETWEEN SOURCE AND DETECTOR}

The cross correlation functions are defined using equations like Eqs. 5.1 and 5.3, but the two functions of the product in the integrand are different functions say $Y$ or $Z$. Thus, there are three cross correlation functions involving the three signal channels: $\mathrm{XY}, \mathrm{XZ}$, and $\mathrm{YZ}$. The first two will be discussed in this section and $\mathrm{YZ}$ in the next section. The time domain equivalent of the correlation between the source and detector is the randomly pulsed neutron measurement (Ref. 10) where the time distribution of pulses after a pulse in the ${ }^{252} \mathrm{Cf}$ source channel is measured.. Unlike the auto correlation function, the cross correlation function is not necessarily symmetric in time $\tau$. The right half of the cross correlation function between each of the detectors and the ${ }^{252} \mathrm{Cf}$ source is the same time distribution obtained from a pulsed neutron measurement. In fact, the original reason for putting ${ }^{252} \mathrm{Cf}$ in an ionization chamber in 1968 was to replace a Cockroft Walton accelerator that was used for pulsed neutron measurements (differential die away) with enriched uranium metal in the mid 1960s. The right half of the cross correlation function provides the same data as an accelerator being used to make neutron pulses after which the time dieaway 
of the neutron population is measured. With an accelerator making pulses, a detector is used to measure the time response or decay between pulses. A simplified illustration of the processing for the cross correlation function is given in Appendix D for a single data block. A typical cross correlation function for positive $\tau$ between one detector and a ${ }^{252} \mathrm{Cf}$ source is shown in Fig. 7.1 for an annular casting with a source on one side and a detector on the other (180 degrees from the source as shown in Fig. 1.4). This is a time of flight transmission measurement through the casting or container. Fig. 7.1 has several interesting features. The time of ${ }^{252} \mathrm{Cf}$ fission (feature a of Fig. 7.1) occurs near $t=0$. The directly transmitted gamma rays appear first in time at the detector, and this is indicated by the feature (b) on Fig. 7.1 Because the uranium of the casting is directly between the source and detector, the directly transmitted prompt gamma rays are attenuated considerably by the high density uranium metal. In addition, they are also delayed slightly. There is a time lapse between the directly transmitted gamma rays and subsequent particles which is larger for greater distances between the source and the detector. The next particles to arrive at the detector are the directly transmitted neutrons and scattered gamma rays illustrated by feature (c) on the figure. The next particles that arrive at the detector are scattered (initial and fission neutrons and gamma rays (feature (d) of Fig. 7.1), and then finally neutrons and gamma rays from later induced fission indicated by (e) on Fig. 7.1. Some of these divisions are not distinct in time but overlap. The time decay of the neutrons from this measurements is the same as that from the so called differential dieaway measurement. At long times, $>500 \mathrm{~ns}$, in this measurement, the slow decay $(f)$ will reach the background level indicated by feature $(g)$ on Fig. 7.1. Since no neutrons or gamma rays from the source or their progeny can occur before the time of ${ }^{252} \mathrm{Cf}$ fission, the left half of the correlation function $(\tau<0)$ is just background and is not shown on this figure.

If a measurement is performed at the same source-detector distance in air as with the casting or container between the source and detector, the following relationship may hold

$I(t)=I_{0}(t) \exp \left[-\sum x\right]$

where $I(t)$ is the flux at the detector at time $t$ with the container between the source and detector, $\mathrm{I}_{\mathrm{o}}(\mathrm{t})$ is the flux without the container, $\sum$ is the removal cross section and $\mathrm{x}$ is the material thickness. This relationship can sometimes be used to identify the material and its thickness between the source and detector. Of course this works best for thin samples equidistant between the source and detector where multiple scattering corrections and neutron scattering to lower energy effects are small. Measurements have been performed using Eq. 7.1 to confirm the presence of $\mathrm{Al}$ and its thickness in B54 trainers returned to Y-12 from the military (Ref. 13). Also, these type measurements have been used to identify $\mathrm{Be}, \mathrm{C}, \mathrm{Pb}, \mathrm{Bi}$, and other materials between the source and detectors (Ref. 14). A plot of this crude approximation to $\sum \mathrm{x}$ for this casting is given in Fig. 7.2 and can be used as another feature for identification. This plot gives this average value for gamma rays and primarily neutrons at later times as a function of time. For times before $12 \mathrm{nsec}, \sum \mathrm{x}$ is positive indicating attenuation but for times greater than $12 \mathrm{nsec}, \Sigma \mathrm{x}$ changes sign because induced fission production becomes larger than attenuation.

\subsection{CROSS CORRELATION FUNCTION BETWEEN DETECTORS}


Analogous processing of time signals for a pair of detector signals [Y(t) and $\mathrm{Z}(\mathrm{t})]$ that was described in Section 7.0 produces a cross correlation function between detectors. If the cross correlation function is between two detectors, this is equivalent to a two detector Rossi- $\alpha$ measurement where the time distribution of pulses in the second detector after a pulse in the first detector is measured. Thus, the right half of this correlation function is the equivalent of the conventional two detector Rossi- $\alpha$ measurement. However, by delaying one of the detector signals [detector 2] in the Rossi- $\alpha$ measurement both the left and right side of the correlation function between two detectors can be obtained. In fact, cross correlation measurements of this type have been reported (Ref. 8). The time decay obtained by this measurement is the same as in a differential dieaway measurement with a pulsed neutron generator or the ${ }^{252} \mathrm{Cf}$ source ionization chamber and is related to the amount and configuration of fissile material in the system. A typical cross correlation function between two detectors is shown in Fig. 8.1 with the following features: (a) symmetric distribution about $\mathrm{t}=0$, (b) time decay, and (c) background. In general, the cross correlation function is not the same for positive lags as it is for negative lags, but for certain cases involving detectors symmetrically located in symmetric systems, it is symmetric in time. A simplified illustration of the processing for the cross correlation function is given in Appendix D.

\subsection{MULTIPLICITIES}

The multiplicities, the number of times $\mathrm{m}$ pulses occur in a data block, are obtained for each channel. The total multiplicity for all channels is also obtained by treating the sum of all detector channels $(2 \& 3)$ as an additional data stream. The distribution functions for some of these channels are plotted and compared to that predicted for random counting corrected for the dead time obtained from the auto correlation function. The multiplicity, $\mathrm{M}$, will always be larger than $\mathrm{M}_{2}$ and $\mathrm{M}_{3} . \mathrm{M}_{1}$ for the source channel can be used to verify proper operation of the source channel detection system and is shown in Fig. 9.1. The number of blocks of data with a given number of counts in a block are plotted vs $\mathrm{m}$. The spontaneous fission process in the ${ }^{252} \mathrm{Cf}$ is random and thus the measured multiplicities for this channel agree with those calculated for random events corrected for the dead time obtained from the auto correlation function of this channel. Multiplicities for detector channel 2 are plotted in Fig. 9.2 and the measured values are only slightly larger than random counting, but significant. A typical plot for $\mathrm{M}$ is shown in Fig. 9.3 for sum of detector channels. The multiplicities obtained from the sum of detectors (Fig. 9.3) are considerably larger than that for a single detector and are as high as order 10. For comparison purposes, the values should be expressed in terms of probabilities so that comparisons made do not depend on the number of blocks in a measurement. From these multiplicities, the

Feynman variance can be obtained as $\left(\overline{c^{2}}-\bar{c}^{2}\right) / \bar{c}$ where $\mathrm{c}$ is the number of counts in a data block.

The ratios, triples/singles, quadruples/singles, quintuples/singles are plotted as a function of the doubles/singles ratio in Fig. 9.4. To show the usefulness of this type of plot, data have been included for the casting with no moderator around it. Since there is less fission without 
moderator, the ratios are generally smaller than with moderator. For higher order ratios (greater than 6/1) the statistical uncertainty is large. The ratios for the casting with 3 in. moderator are given in Table 9.1.

\section{Table 9.1 Ratios of Combined Multiplicities For All Detectors For A HEU-Metal Casting}

\begin{tabular}{|c|c|}
\hline Ratio To Singles & Value \\
\hline \hline $2 / 1$ & $1.92 \mathrm{E}-01$ \\
\hline $3 / 1$ & $2.74 \mathrm{E}-02$ \\
\hline $4 / 1$ & $3.87 \mathrm{E}-03$ \\
\hline $5 / 1$ & $5.49 \mathrm{E}-04$ \\
\hline $6 / 1$ & $8.03 \mathrm{E}-05$ \\
\hline $7 / 1$ & $1.24 \mathrm{E}-05$ \\
\hline $8 / 1$ & $1.95 \mathrm{E}-06$ \\
\hline $9 / 1$ & $2.73 \mathrm{E}-07$ \\
\hline $10 / 1$ & $2.27 \mathrm{E}-08$ \\
\hline
\end{tabular}

\subsection{AUTO POWER SPECTRAL DENSITIES}

The auto power spectral density is the Fourier decomposition of a single signal into its frequency components. An example of this is a Fourier analysis of a voice signature that can be used for identification. Frequency analysis signatures are usually obtained as follows (Ref. 15). The detector signals are accumulated in time bins to form blocks of data. Standard Fast Fourier Transforms (FFT) are applied to a data block to produce real and imaginary components of the transform. Complex multiplications of the transform with the complex conjugate of itself produces an estimate of the auto power spectral density which is a real number with no imaginary part. The process is repeated on the subsequent block of data and the results are averaged with the first. This process continues until the desired statistical precision is obtained. Until 1996, all NWIS-type frequency analysis was performed in this manner. With the new NWIS processor, the auto power spectral density $\left(\mathrm{G}_{\mathrm{ii}}\right)$ is obtained by Fourier transforming the auto correlation function $\left(\mathrm{AC}_{\mathrm{ii}}\right)$. This is theoretically and numericaily equivalent to the previous processing of data for frequency analysis signatures. The auto power spectral densities (APSD) for the source and one of the two detectors are shown in Fig. 10.1 and 10.2. For systems with low neutron multiplication factor and low detection efficiency the APSDs are constant with frequency. The APSD of the source, $G_{11}$, is normalized to 1 . The APSD of the detectors are sensitive to count rate and all contributions to the count rate such as background, spontaneous fission, induced fission, etc. contribute to $G_{22}$ and $G_{33}$. Again, the APSD of the source channel $G_{11}$ describes the operation of the source detection system. 


\subsection{CROSS POWER SPECTRAL DENSITIES}

The cross power spectral density is a measure of the common information in two signals. In this case, the two data streams from two detectors (X, Y; X,Z; or Y,Z) are usually sampled into separate time bins to form blocks of data. The blocks are fast Fourier transformed. Each transformed block consists of real and imaginary parts. The transformed blocks are cross multiplied (the complex conjugate of one with the other, i.e., $\mathrm{X}^{*}$ with $\mathrm{Y}$ rather than $\mathrm{X}^{*}$ with $\mathrm{X}$ as in the APSD) producing an estimate of the real and imaginary parts of the CPSD XY. Additional blocks are sampled and averaged with the previous data to obtain better estimates of the real and imaginary parts of the CPSD XY. This process continues until the desired statistical precision is obtained. In all NWIS frequency analysis measurements until 1996, this method was used. With the new NWIS processor the CPSD XY is obtained by Fourier transforming the cross correlation function. The CPSD has the interesting property that for frequency greater than zero the background does not contribute to the CPSD. This was first realized by Stegeman and Numuro (Refs. 16 and 17) in 1966. They applied the CPSD to eliminate problems with the background of the APSD which, in many cases for subcritical fissile measurements, obscured the reactor physics information. The real and imaginary parts of the CPSD define the phase between signals. Time delays are represented by phase in frequency analysis ( 360 degrees phase at the sampling frequency is one sampling interval). Large time delays between signals mean large phase.

The CPSD between the detection channel and the source measures the common information in the two signals, where the common information in the detector comes from induced fission in the system by neutrons from ${ }^{252} \mathrm{Cf}$. The CPSD between the two detectors measures the common information because both detectors are counting particles from ${ }^{252} \mathrm{Cf}$ fission and induced fission in the system. Typical plots of the magnitude of the CPSDs are shown in Figs. 11. 1 and 11.2.

Another useful plot of the CPSD data is the imaginary (ordinate) vs the real (abscissa). One such plot, between the source and a detector, is shown in Fig. 11.3. By removing the frequency response of the detectors and processing systems obtained from a calibration measurement as described in section 4.0 , the transfer function of the fissile system can be estimated. In this polar plot (Fig. 11.3), the number of times the curve crosses the ordinate or abscissa is the number of poles in the transfer function. For example, Fig. 11.3 has four crossing of the ordinate or abscissa, thus four poles or four different decay constants to represent the time decay of the neutrons and gamma rays. Usually for symmetric locations of the detectors with respect to the source and fissile system, the imaginary part of the CPSD between detectors is near zero compared to the real part because there are no time delay differences between detectors. This not true for CPSDs between the detectors and the ${ }^{252} \mathrm{Cf}$ source ionization chamber, $\mathrm{G}_{12}$ and $\mathrm{G}_{13}$.

\subsection{COHERENCES}

The coherence, $\gamma_{i j}^{2}$, is another useful signature. It is the fraction of common information in two channels, it is real; and it varies between 0 and 1 . The coherence is defined as 
$\gamma_{i j}^{2}=\frac{\left|G_{i j}\right|^{2}}{G_{i i} G_{i j}}$.

Measurements are readily performed with NWIS where $\gamma_{i j}^{2}$ is much lower than $10^{-6}$. Typical values are given in Fig. 12.1 and 12.2. The coherence usually decreases with frequency since there is less correlated signal at high frequency for the CPSDs.

\subsection{RATIO OF SPECTRAL DENSITIES}

In previous time domain measurements (Ref. 18), it was realized that a measured parameter independent of the detection efficiency could be obtained from measurements if both cross correlation functions between detectors and between detectors and the ${ }^{252} \mathrm{Cf}$ source were measured simultaneously. The frequency analysis equivalent of this early time analysis expression is the following ratio of spectral densities.

$$
R_{123}(\omega)=\frac{G_{12}^{*}(\omega) G_{13}(\omega)}{G_{11}(\omega) G_{23}(\omega)} .
$$

where * means complex conjugation. In this expression detection efficiencies appear in both the numerator and denominator but cancel much in the same way as in beta-gamma coincidence counting methods to obtain the neutron flux in fission reactors. This has lead to a signature which is easily reproduced. Measurements made 10 years apart with uranium metal cylinders yielded the same value of $R(\omega)$ even though different sources and detectors were used (Ref. 19). In fact, measurement with detectors sensitive only to gamma rays yields the same result as that for detectors sensitive only to neutrons. This ratio is also independent of uncorrelated background which also makes it more useful. A plot of this ratio for the casting is shown in Fig. 13.1 where the ratio decreases with frequency until it is near 0 at $100 \mathrm{MHz}$. The magnitude of the ratio of spectral densities can be shown theoretically to obey the following relationship,

$|R(\omega)|^{2}=\gamma_{12}^{2} \cdot \gamma_{13}^{2} / \gamma_{23}^{2}$

\subsection{FREQUENCY ANALYSIS SIGNATURES WITHOUT TRANSMITTED GAMMA RAYS}

An option in the data processing is to remove the transmitted gamma ray peak from the cross correlation functions $\mathrm{AC}_{12}$ and $\mathrm{AC}_{13}$, thus producing CPSD $\mathrm{G}_{12}$ and $\mathrm{G}_{13}$, and coherences $\gamma_{12}^{2}$ 
and $\gamma_{13}^{2}$ which depend primarily on secondary gamma rays and neutrons. This transmitted gamma removal approach is particularly useful where these gamma rays are the predominant part of the signal. This removal emphasizes the signal from induced fission and can be useful. This approach preferentially reduces the high frequency content because the prompt gamma rays from ${ }^{252} \mathrm{Cf}$ fission arrive first as a sharp pulse at the detector. This gamma ray peak contributes at all frequencies but there are not many other contributions at high frequency.

\subsection{MONTE CARLO TRANSPORT CALCULATIONS OF SIGNATURES}

All time and frequency analyses signatures that have been described in the previous sections can be calculated using the MCNP-DSP (Refs. 20 and 21) Monte Carlo transport code. This code calculates the signatures in the same ways as they are obtained by the new NWIS processor but as yet does not include dead time affects on the calculation of the signatures. This code (a major modification of MCNP) is a coupled neutron-gamma ray transport code that tracks neutrons and gamma rays from the source into the system and to the detectors to provide the sequences of detector pulses that form blocks of data from which the time and frequency analysis parameters are obtained. MCNP-DSP uses probability distributions rather than average values of quantities like the number of neutrons per fission in order to better treat the physical processes on an event by event basis. In this way, signatures that depend on the higher moments of the particle population are more properly calculated. In MCNP-DSP, detection processes for detectors that are sensitive to neutron and/or gamma ray scattering are approximated. A detection event occurs based on energy deposited above an energy threshold rather than modeling the light production and light detection processes in the Monte Carlo code. This results in considerable savings in computer calculation time and works quite well for plastic or other organic proton recoil scintillators sensitive to both neutrons and gamma rays and for inorganic scintillators sensitive to gamma rays.

\subsection{CONCLUSIONS}

The new high speed NWIS data acquisition processor that is capable of handling $1 \mathrm{GHz}$ sampling rates allows good resolution measurements of nuclear weapons/components. A variety of signatures have been developed both in the time and frequency domains. Their shape and magnitude contain features that provide information on the systems interrogated. This large number of signatures provides a wide variety of methods for comparison of fissile items in containers. All signatures are not independent but exhibit interesting features that can be used to distinguish weapons components. If background conditions were constant, all of the signatures would be useful. When the background varies considerably, those signatures involving the detector and the source are useful, particularly cross power spectral densities $G_{12}$ and $G_{13}$. Automated pattern recognition methods are not yet fully developed but can be used for comparison of signatures. 


\section{APPENDIX A: OTHER USES OF THIS METHODOLOGY}

In addition to the application to the identification of nuclear weapons/components, this system has a wide variety of applications which are briefly described in this section. The high sensitivity of the measured parameters in this method to changes for some of the measured parameters makes it particularly useful. This sensitivity is described in Ref. 22. This type of system can be used for the nuclear criticality safety to determine the subcritical neutron multiplication factor for inplant configurations of fissile materiais. A related application for the nuclear criticality safety is to use the data from this system to validate transport theory calculational methods for nuclear criticality safety, which is required by DOE orders. These two applications are illustrated in Ref. 23 where this methodology was applied to a vault at the Oak Ridge Y-12 Plant for storage of highly enriched uranium.

Another recent new use of the method is to characterize nuclear material deposits in a shutdown diffusion plant. This measurement method was adapted to image the deposit, obtain the hydrogen-to-uranium ratio, and the mass of the deposit, all of which are of interest for nuclear criticality safety. This characterization was performed without a calibration other than an empty pipe. This information was extremely useful for obtaining permission to proceed with deposit removal. This deposit characterization with NWIS was verified when the Deposit Removal Program at the East Tennessee Technology Park drilled into the pipe at the K-29 Plant for visual observation and found a deposit adjacent to the top of the inside of the pipe as measured by NWIS.

This methodology can be used for conventional nondestructive assay of fissile materials with calibration standards and, in many cases, will be more sensitive to changes than conventional nondestructive assay now deployed. Another use of this methodology is to obtain fingerprints of limited or unusual fissile material and to use these fingerprints to track these materials in storage or until they can be put into a form for which calibration standards exist. Of course, this implementation would be ideal if the fingerprint was obtained at the time of initial packaging.

In addition to obtaining a fingerprint of a static sample as described above, this methodology could be used to track dynamic nuclear material processing such as a pit conversion, recasting of fissile materials. For example, detectors and $\mathrm{a}^{252} \mathrm{Cf}$ source could be deployed around a casting facility for recasting HEU or around a Pu calciner which converts oxide to Pu metal or around dissolvers for fuel elements for reprocessing. A dynamic fingerprint is obtained to characterize the process and deviations from normal operation could be detected that may be related to diversion or safety such as double batching of a casting. It also has applications for fuel fabrication (including Mox fuel) and spent fuel storage/transportation. A dynamic measurement was performed in 1987 as part of a cooperative program with the Power Reactor and Fuel Development Corporation of Japan (Ref. 24). In this experiment, a solution of uranyl (93.2 wt\% of ${ }^{235} \mathrm{U}$ ) was drained from a tank in $\sim 60 \mathrm{sec}$, and these noise analysis measurements performed to obtain the frequency analysis signatures described here as the tank drained. As the tank drained, the ratio of spectral densities was interpreted every 6 seconds to obtain "on-line" the neutron multiplication factor $(k)$ which varied from $k=0.96$ when the tank was full to $k=0.3$ when the tank was near empty. 
The above general applications can be further subdivided into a variety of subcategories of implementation: dismantlement of nuclear weapons, tracking and monitoring of

weapons/components in deployment or storage as well as into the areas for disposal of materials. In generai, this methodology has a broad range of applications for fissile material, some of which are mentioned here and in other experiments or measurements where one nsec second timing of detection events is required. 


\section{APPENDIX B: ALTERNATE SOURCES}

An associated particle sealed tube neutron generator (APSTNG) is an alternate source for NWIS (Ref. 25). The APSTNG source has several advantages. It can be turned off when not in use, and this simplifies handling and transportation of the source. Since the APSTNG source produces 14-MeV neutrons, the source is more penetrating for materials with significant amounts of hydrogen. Another advantage is that the direction of the neutrons is defined, and this may be useful since it could be aimed at the weapons component or fissile material under interrogation, especially for material in containers. Since the pulses from the $\alpha$-particle detector of APSTNG are associated with neutrons that go in the right direction, it may be an advantage for materials in large containers. A consequence of the fact that the DT source emits one particle is that if correlations are measured between the detectors, they will be from the fissile material and not the source. This extends the range of the cross power spectral density and coherence between detectors to zero and the ratio of spectral densities from near 0 at delayed critical to infinite when no fissile material is present. Thus, this source may be more useful for assaying small amounts of fissile material.

APSTNG has several disadvantages. This first is that it is more complicated and more difficult to operate than ${ }^{252} \mathrm{Cf}$. APSTNG contains tritium and requires high voltage $(\sim 120 \mathrm{kV})$ to operate, both of which are hazardous. Alpha particle detection methods with this source must detect all of the alpha particles, or possible correlated source particles are lost. The advantages and disadvantages of the APSTNG and ${ }^{252} \mathrm{Cf}$ sources are summarized in Table B.1. The NWIS processor could be used with conventional repetitively pulsed (fixed frequency) neutron generators and in this use an electrical or radio frequency pulse that corresponds to the particle pulse from the neutron generators would be input to channel 1 .

Table B.1. Advantages and disadvantages of ${ }^{252} \mathrm{Cf}$ and APSTNG sources

\begin{tabular}{|c|c|c|}
\hline Source & Advantages & Disadvantages \\
\hline${ }^{252} \mathrm{Cf}$ & $\begin{array}{l}\text { 1. Simple } \\
\text { 2. Reliable } \\
\text { 3. Small } \\
\text { 4. All source events detected }\end{array}$ & $\begin{array}{l}\text { 1. Cannot turn off-small } \\
\text { radiation dose in handling } \\
\text { 2. More than one neutron per } \\
\text { source event }\end{array}$ \\
\hline APSTNG & $\begin{array}{l}\text { 1. Directional } \\
\text { 2. Can be turned off } \\
\text { 3. More penetrating neutrons for } \\
\text { hydrogen-bearing materials } \\
\text { 4. Extends the range of measured } \\
\text { CPSDs, coherences, and } \\
\text { ratios of spectral densities }\end{array}$ & $\begin{array}{l}\text { 1. Complicated } \\
\text { 2. Large, heavy } \\
\text { 3. Tritium is radioactive } \\
\text { 4. Not all } \alpha \text { particles hitting the } \alpha \\
\text { detector of source are detected } \\
\text { 5. Limited life }\end{array}$ \\
\hline
\end{tabular}




\section{APPENDIX C: AUTO CORRELATION FUNCTION PROCESSING}

Consider a simple example of a shift register (Type I time analyzer) being used for a Rossi- $\alpha$ measurement. For illustrative purposes, suppose the shift register has the following pulse sequence in its 8 time bins and no other pulses after these time bins.

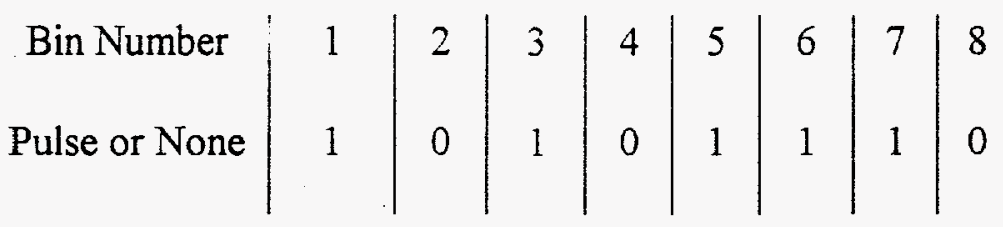

Pulses present are represented by 1 . Either pulses are present or they are not. In a Type I time analyzer (time analyzer where no pulses are lost, i.e. zero dead time), pulses are shifted to the left and each time a pulse is present in time bin one, subsequent pulses are stored according to their time after channel 1 . This continues for the 8 shifts to the left until the following time distribution $5,2,3,1,2,1,1,0$ is finally obtained. The summation in the correlation function (Eq. 5.3) for this same 8 bin sequence is illustrated in Table C.1.

TABLE C.1. COMPUTATION OF SUMMATION $\sum_{k=0}^{N-1-\tau} \mathrm{X}_{k} \mathrm{X}_{k+\tau}$ FOR BLOCK OF 8 TIME BINS

\begin{tabular}{|c|c|c|c|c|c|c|c|c|c|c|c|}
\hline \multirow{2}{*}{$\begin{array}{l}\text { LAG } \\
\tau=0\end{array}$} & \multicolumn{8}{|c|}{ SEQUENCES } & \multicolumn{2}{|c|}{ RESULT } & \multirow{2}{*}{$\begin{array}{l}\text { COINCIDENCE BETWEEN SHIFTED } \\
\text { BLOCK AND ITSELF }=\sum \mathbf{x}_{\mathrm{k}} \mathbf{x}_{\mathrm{k}+\tau} \\
\text { Coincidence between unshifted block and itself }\end{array}$} \\
\hline & 1 & $\mathrm{O}$ & 1 & o & 1 & 1 & 1 & 0 & $\rightarrow$ & 5 & \\
\hline$\tau=1$ & 0 & 1 & 0 & 1 & 1 & 1 & 0 & 0 & $\rightarrow$ & 2 & Coincidence between $x_{k}$ and $x_{k+1}$. \\
\hline$\tau=2$ & 1 & 0 & 1 & 1 & 1 & 0 & 0 & o & $\rightarrow$ & 3 & Coincidence between $x_{k}$ and $x_{k+2}$. \\
\hline$\tau=3$ & $\mathrm{O}$ & 1 & 1 & 1 & $\mathrm{O}$ & o & o & 0 & $\rightarrow$ & 1 & Coincidence between $x_{k}$ and $x_{k+3}$. \\
\hline$\tau=4$ & 1 & 1 & 1 & O & $\mathrm{O}$ & o & 0 & 0 & $\rightarrow$ & 2 & Coincidence between $x_{k}$ and $x_{k+4}$. \\
\hline$\tau=5$ & 1 & 1 & 0 & $\mathrm{O}$ & $\mathrm{O}$ & $\mathrm{O}$ & $\mathrm{O}$ & o & $\rightarrow$ & 1 & Coincidence between $x_{k}$ and $x_{k+5}$. \\
\hline$\tau=6$ & 1 & $\mathrm{O}$ & 0 & $\mathrm{O}$ & $\mathrm{O}$ & $\mathrm{O}$ & $\mathrm{O}$ & $\mathrm{O}$ & $\rightarrow$ & 1 & Coincidence between $x_{k}$ and $x_{k+6}$. \\
\hline$\tau=7$ & o & 0 & o & $\mathrm{o}$ & $\mathrm{O}$ & $\mathrm{o}$ & $\mathrm{o}$ & o & $\rightarrow$ & 0 & Coincidence between $x_{k}$ and $x_{k+7}$. \\
\hline
\end{tabular}

The result of $\sum_{k=0}^{N-1-\tau} X_{k} X_{k+\tau}$ is the same as that obtained above in the shift register for a single detector Rossi- $\alpha$ measurement. The difference between a Rossi- $\alpha$ measurement and the summation in the auto correlation function is that the Rossi- $\alpha$ shift register processes a continuous stream of pulses that come in from the right and are shifted to the left. When a pulse 
is in time bin or register 1 at the far left, subsequent pulses are stored in their appropriate time bins. This process continues until the final distribution of pulses is obtained. For the correlation function, summation of a finite sample of data ( 8 bins for this case) is used rather than a continuous stream of pulses sorted into 8 bins. The unbiased linear correlation function accounts for finite block size effects. 


\section{APPENDIX D: CROSS CORRELATION FUNCTION PROCESSING}

For this type of correlation, consider two 8 bin blocks of data $Y$ and $Y$ and no subsequent data in a two channel shift register (Type I time analyzer).

\begin{tabular}{c|l|l|l|l|l|l|l|l} 
Bin Number & 1 & 2 & 3 & 4 & 5 & 6 & 7 & 8 \\
$\mathrm{X}(\mathrm{t})$ & 1 & 0 & 1 & 0 & 1 & 1 & 1 & 0 \\
$\mathrm{Y}(\mathrm{t})$ & 1 & 1 & 0 & 1 & 0 & 0 & 1 & 1
\end{tabular}

Pulses in both channels are shifted to the left, and every time there is a pulse in channel $\mathrm{X}$ in the first bin the value of $Y$ is stored and the results summed when the 8 shifts are done. The following sequence is obtained after the 8 shifts $2,4,2,2,1,1,1,1$. Now consider the summation $\sum X_{k} Y_{k+\tau}$ for this example as illustrated in the following Table D.1. The data of Table D. 1 that corresponds to the shift register example is that for $\tau$ greater than or equal to 0 .

Table D.1. COMPUTATION OF SUMMATION $\sum_{k=0}^{N-1-\tau} X_{k} Y_{k+\tau}$ FOR BLOCK OF 8 TIME BINS

\begin{tabular}{|c|c|c|c|}
\hline Lag & Sequences & Result & Coincidence Between Shifted Block and Itself \\
\hline$\tau=-7$ & 00000000 & 0 & Coincidence between $X_{k}$ and $Y_{k-7}$ \\
\hline$\tau=-6$ & 10000000 & 1 & Coincidence between $X_{k}$ and $Y_{k-6}$. \\
\hline$\tau=-5$ & 11000000 & 2 & Coincidence between $X_{k}$ and $Y_{k-5}$. \\
\hline$\tau=-4$ & 11000000 & 2 & Coincidence between $X_{k}$ and $Y_{k-4}$. \\
\hline$\tau=-3$ & 01010000 & 2 & Coincidence between $X_{k}$ and $Y_{k-3}$. \\
\hline$\tau=-2$ & 10010000 & 2 & Coincidence between $X_{k}$ and $Y_{k-2}$ \\
\hline$\tau=-1$ & 01010000 & 2 & Coincidence between $X_{k}$ and $Y_{k-1}$ \\
\hline$\tau=0$ & 10000010 & 2 & Coincidence between $X_{k}$ and $Y_{k}$ \\
\hline$\tau=1$ & 10100110 & 4 & Coincidence between $X_{k}$ and $Y_{k+1}$ \\
\hline$\tau=2$ & 00001100 & 2 & Coincidence between $X_{k}$ and $Y_{k+2}$ \\
\hline$\tau=3$ & 10001000 & 2 & Coincidence between $X_{k}$ and $Y_{k+3}$ \\
\hline$\tau=4$ & 00100000 & 1 & Coincidence between $X_{k}$ and $Y_{k+4}$ \\
\hline$\tau=5$ & 00100000 & 1 & Coincidence between $X_{k}$ and $Y_{k+5}$ \\
\hline$\tau=6$ & 10000000 & 1 & Coincidence between $X_{k}$ and $Y_{k+6}$ \\
\hline$\tau=7$ & 10000000 & 1 & Coincidence between $X_{k}$ and $Y_{k+7}$ \\
\hline
\end{tabular}

For $\tau \geq 0$, this result is the same as for the shift register time analyzer given above in text. 


\section{APPENDIX E: NON INTRUSIVE USE FOR IDENTIFICATION OF NUCLEAR WEAPONS}

This appendix illustrates how this system can be used nonintrusively (Refs. 26, and 27). The use of NWIS signatures to confirm nuclear weapons components in containers is illustrated by measurements with many components of the same type. Reference signatures were obtained for a single container with a known component, and the signature for other containers was compared to the reference. A photograph of a cart portable version of NWIS is given in Fig. E.1. The set of 19 time and frequency analyses signatures were compared. A nonintrusive display of the comparisons can be made by dividing the signature of the item in a container by the reference signature. Not all of the 19 time and frequency analysis signatures can be presented in this appendix. The primary signatures illustrated are the CPSDs between the detectors and ${ }^{252} \mathrm{Cf}$ source. These CPSDs are insensitive to background (for frequency, $\omega>0$ ) and have advantages for storage warehouse applications and tracking components through the first stage of dismantlement. Measurement with the reference unit establish the statistical precision of the signatures. The variation of the signatures from container to container is established by measurements with several containers. This variation is a result of packaging, location of the units in the containers, and the variation in the placement of the source and detectors. Once these variations are established, an acceptable band of signatures can be defined that can be used to characterize the item in the containers. Typical ratios of CPSDS $G_{12}$ and $G_{13}$ to like signatures for the reference unit as a function of frequency are given in Fig. E. 1 as the solid lines. Also shown on Fig. E. 1 are the acceptable bands for both $G_{12}$ and $G_{13}$ defined by many measurements for containers with the same weapons component. The bounds of the bands are the maximum and minimum ratios from the set from measurements on many containers with this same component. The measurement time for these confirmations is $\sim 2 \mathrm{~min}$. Clearly, the solid lines show that the measurements fall within the set and this item belongs to the set. To illustrate how another type component in the same sized container falls outside the set, consider the CPSD 12 between one of the detectors and the source. The ratio of CPSD 12 to the reference for this blind test with another weapons component in the same size container is shown in Fig. E.2 and clearly shows that this item is different from the set.

Another way to make comparisons of signatures is using pattern recognition algorithms. One illustrated here to compare signatures $\mathrm{G}_{12}$ for these measurements was the Mahalanobis method, which compares the absolute deviation (in units of standard deviation) of a signature at a frequency point for each data point (Ref. 28). Where the signature consists of 256 frequency points $(\mathrm{N})$, as with these data, the Mahalanobis distance,

$$
\mathrm{M}^{\prime}=\frac{1}{N} \sum_{i=1}^{N}\left|x_{i}-x_{j}\right| / \sigma_{i}
$$

where $\mathrm{j}$ designates the reference. Thus, it is the average absolute deviation expressed in units of standard deviation and thus is dimensionless. For exactly repeatable measurements with only statistical errors, the value of $\mathrm{M}^{\prime}$ can be shown to theoretically be $\sim 0.80$. For the set of items verified here the values of $\mathrm{M}^{\prime}$ varied between 0.5 and 1.5 with half the values less than 0.8 and, half greater than 0.8 . For the item that was different from the set of like items, the value of $M$ for 
CPSD 12 is 12 , which differs significantly from the values for the set. Values of $\mathbf{M}^{\prime}$ for the item not in the set compared to the set for the other frequency spectra $G_{22}, G_{33}, G_{13}$, and $G_{23}$ were as high as 20; all clearly determining that this weaporis component was not in the set. The use of the Mahalanobis distance for comparisons allows decisions on comparisons to be made without any display of measured data. 


\section{REFERENCES}

1. J. T. M.halczo and V. K. Paré, "Nuclear Weapons Idertification System," Arms Control and Vonproliferation Technologies, 24-26, Third Quarter 1994

2. 5. T. Mihalczo, T. E. Valentine, and J. K. Mattingly, "Feasibility of Subcriticality and NDA Measurements for Spent Fuel by Frequency Analysis Techniques with ${ }^{2 \varsigma 2} \mathrm{Cf}$," pp. 883-891 in Proceedings of the Topica: Meeting on Nuclear Plant Instrumentation, Control and HumanMachine Interface Technologies, University Park, Pennsylvania, American Nuclear Society, ISBN: 0-89448-610-1; Vol. II, 883-891 (1996).

3. T. E. Valentine, J. T. Mihalczo, and P. E. Koehler, "Calculated NWIS Signatures for Enriched Uranium Metal," Proceedings of the 36th. Annual Meeting of Institute of Nuclear Materials Management, Palm Desert, California, Vol. XXIV, 388-393 (1995).

4. J. E. Breeding, J. A. Mullens, G. W. Turner, T. E. Valentine, J. A. McEvers, J. T. Mihalczo, and T. A. Gafford, "New NWIS Processor For Fissile System Verification," Institute of Nuclear Materials Management Meeting, Phoenix, Arizona, July 20-24, 1997.

5. Sjostrand, N. G., "Measurements on a Subcritical Reactor Using a Pulsed Neutron Source," Arkiv for Fysik, Vol. 11, p. 233, 1956.

6. J. D. Orndoff, "Prompt Neutron Periods of Metal Critical Assemblies," Nuclear Science and Engineering, Vol. 2, p. 450, 1957.

7. J. T. Mihlaczo, "Prompt Neutron Decay and Reactivity Measurements in Subcritical Uranium Metal Cylinders," Nucl. Sci. Eng., 32, 292 (1968).

8. J. T. Mihlaczo, "Prompt Neutron Lifetime in Critical Enriched-Uranium Metal Cylinders and Annuli," Nucl. Sci. Eng., 20, 60 (1964).

9. J. T. Mihlaczo, "The Use of ${ }^{252} \mathrm{Cf}$ as a Randomly Pulsed Neutron Source for Prompt Neutron Decay Measurements," Nucl. Sci. Eng., 41, 296 (1970).

10. J. T. Mihalczo, V. K. Paré, G. L. Ragan, M. V. Mathis, and G. C. Tillett, "Determination of Reactivity from Power Spectral Density Measurements with Californium-252," Nucl. Sci.

Eng., 66, 29-59 (1978).

11. M. J. Paulus, J. T. Mihalczo, T. E. Valentine, J. A. Mullens, J. E. Breeding, T. Uckan, J. K. Mattingly, G. W. Turner, M. C. Smith, and J. A. McEvers, "A Novel Method for Determining Pulse Counting Circuitry Dead Time Using the Nuclear Weapons Inspection System," IEEE, Nucl. Sci. Symp. 1997.

12. M. Edelman, "New Rossi- $\alpha$ Measurement Methods," INR-4/68-15, Karlsruhe (1968). 
13. J. T. Mikalozo, V. K. Paré, E. D. Blakeman, and T. E. Valentine, "NWIS Signatures for Trains: Confirmation at the Oak Ridge Y-12 Plant," Y/WR-202, Institute of Nuclear Matarials Management, Napies, Florida (1996).

14. J. T. Miralczo and N. W. Hill, "Simple Time-of-Flight Transmission Measurement for Incorporation in Nuclear Engineering Curricula," Trans. Am. Nucl. Soc. 14(1), 60-61 (1971).

15. J. S. Bendat and A. G. Piersol, Random Data: Analysis and Measurement Procedure, Wiley-Interscience, New York, 1971.

16. W. Seifritz, D. Stegemamn, W. Vath, "Two Detector Cross Correlation Experiments in the Fast-Thermal Argonaut Reactor Stark," International Symp. On Neutron Noise, Waves, and Pulse Propagation, Gainesville, Florida, February 14-16, 1966.

17. T. Nomura, "Improvement in S/N Ratio of Reactor Noise Spectral Density," Journal of Nuclear Science and Technology (Tokyo), Vol. 2, p. 76, 1965.

18. J. T. Mihalczo, "New Method for Measurement of the Effective Fraction of Delayed Neutrons from Fission," Nucl. Sci. Eng., 46, 147 (1971).

19. J. T. Mihalczo, W. T. King, and E. D. Blakeman, "252 Cf Source-Driven Neutron Noise Analysis," Workshop on Subcritical Reactivity Measurements, Albuquerque, New Mexico Conference 8508/05, August 1985.

20. T. E. Valentine and J. T. Mihalczo, "Validation of the Monte Carlo Code MCNP-DSP," Annals of Nuclear Energy 24, No. 2, 79-98 (1997).

21. T. E. Valentine and J. T. Mihalczo, "MCNP-DSP: A Neutron and Gamma Ray Monte Carlo Calculation of Source-Driven Noise-Measured Parameters," Annals of Nuclear Energy, 23, No. 8, 1271-1287 (1996).

22. J. T. Mihalczo and T. E. Valentine, "Calculational Verification and Process Control Applications Utilizing the High Sensitivity of Noise Measurement Parameters to Fissile System Configuration," Nucl. Sci. Eng., 121, 286-300 (1995).

23. J. T. Mihalczo, T. E. Valentine, and L. D. Phillips, "Subcritical Measurements for a HEU Storage Vault at the Y-12 Plant Using the ${ }^{252}$ Cf-Source-Driven Frequency Analysis Method," Nuclear Safety (1997 in press).

24. J. T. Mihalczo, E. D. Blakeman, G. E. Ragan, E. B. Johnson, and Y. Hachiya, "Dynamic Subcriticality Measurements Using the ${ }^{252}$ Cf-Source-Driven Noise Analysis Method," Nucl. Sci. Eng., 104, 314-38 (1990).

25. J. T. Mihalczo, P. E. Koehler, T. E. Valentine, and L. D. Phillips, "Source Options for Nuclear Weapons Identification System," ORNL/TM-13025 (July 1995). 
26. J. T. Mihaiczo, T. E. Valentine, J. A. Mullens, "Nonintrusive Nuclear Weapons/Coniponent Identification System For Internationai Inspections," 5th International Conference on Nuslear Engineering, Nice, France, May 26-30, 1997.

27. J. T. Mihalczo, T. E. Valentine, J. A. Mullens, "Non Intrusive ${ }^{252}$ Cf-Source-Driven Correlations For Verification of Nuclear Weapons Components in Storage," Russian International Conference on Nuclear Material Protection, Accounting, and Control, Obninsk, Russia, March 9-14, 1997.

28. J. T. Tou and R. C. Gonzalez, "Pattern Recognition Principles," p. 87, 125-127,(1974). 


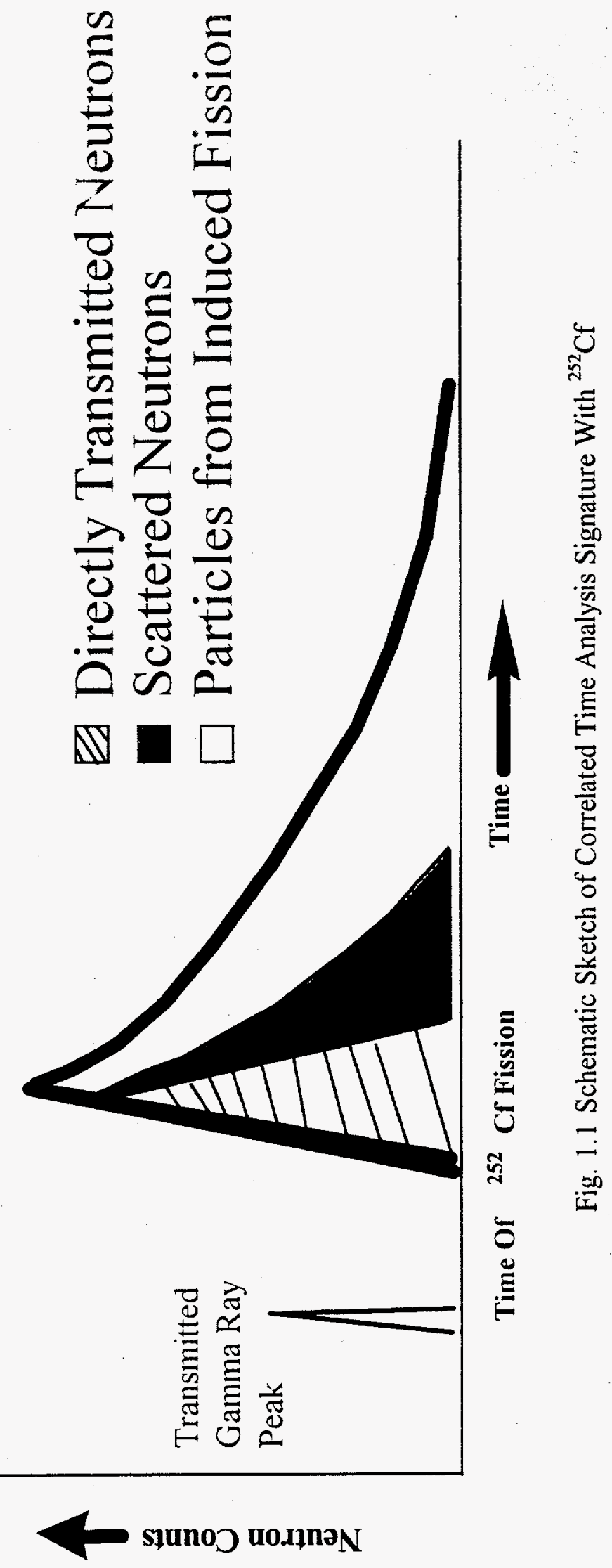




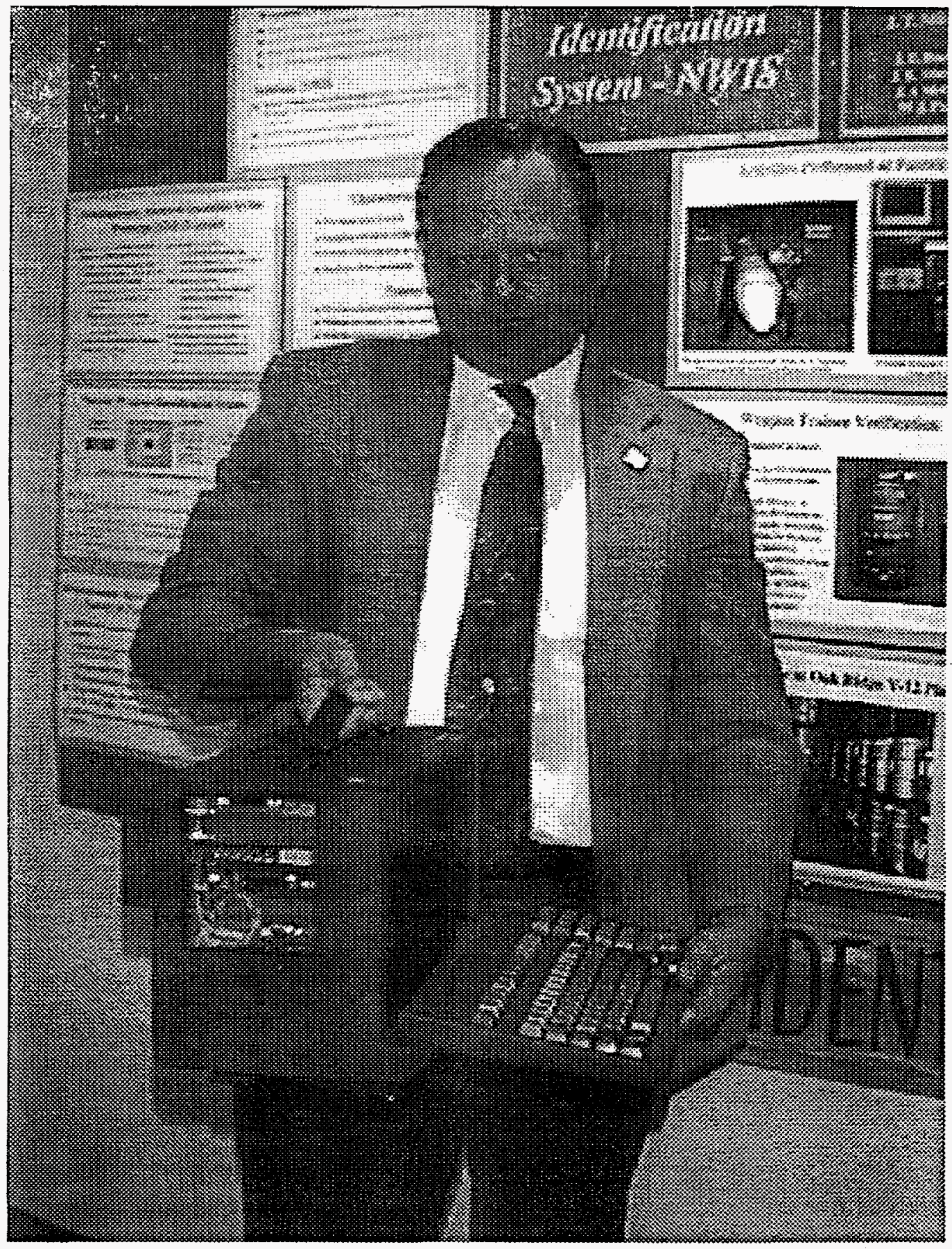

Fig. 1.2. Photograph of a portable version of the NWIS processor 


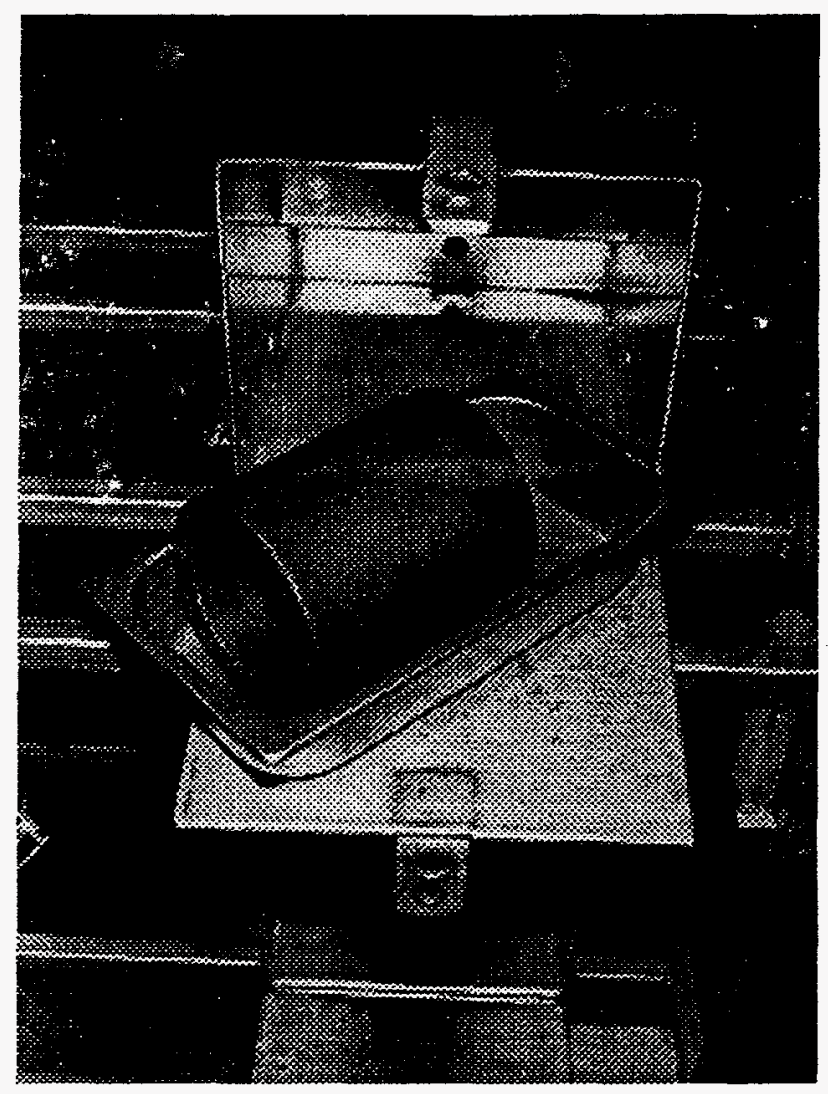

Fig. 1.3A. Photograph of a uranium metal annular casting in a steel tray

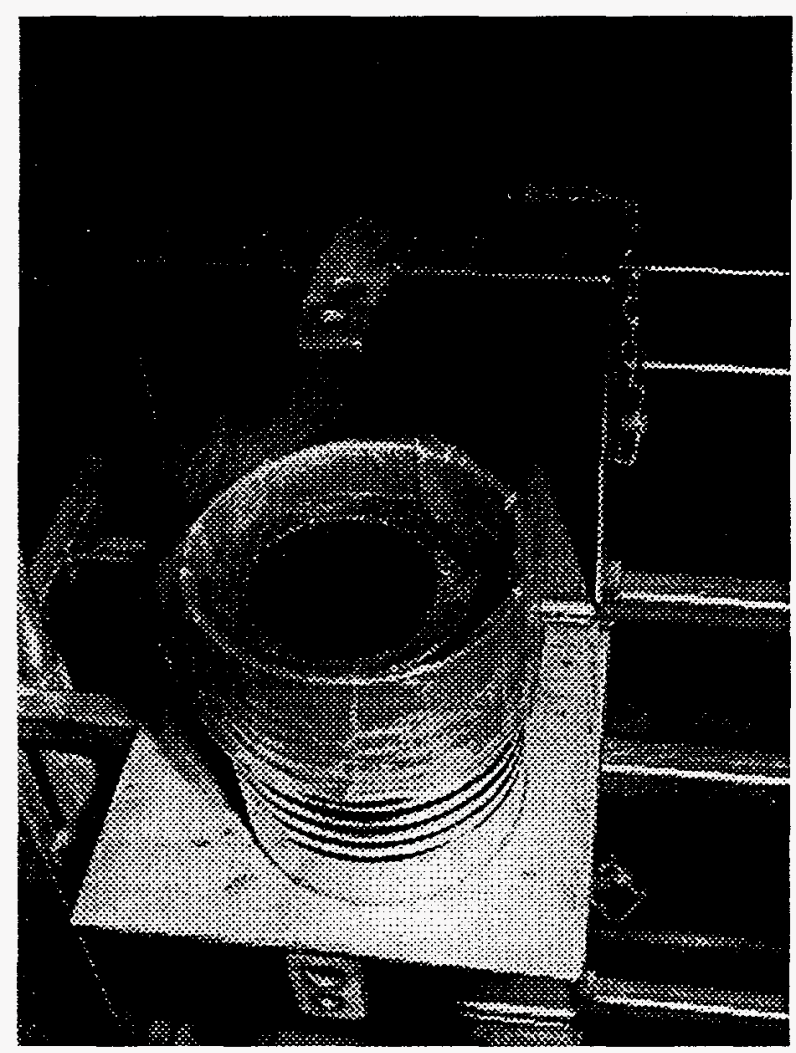

Fig. 1.3B. Photograph of an annular casting in its plastic bag in an unsealed can 


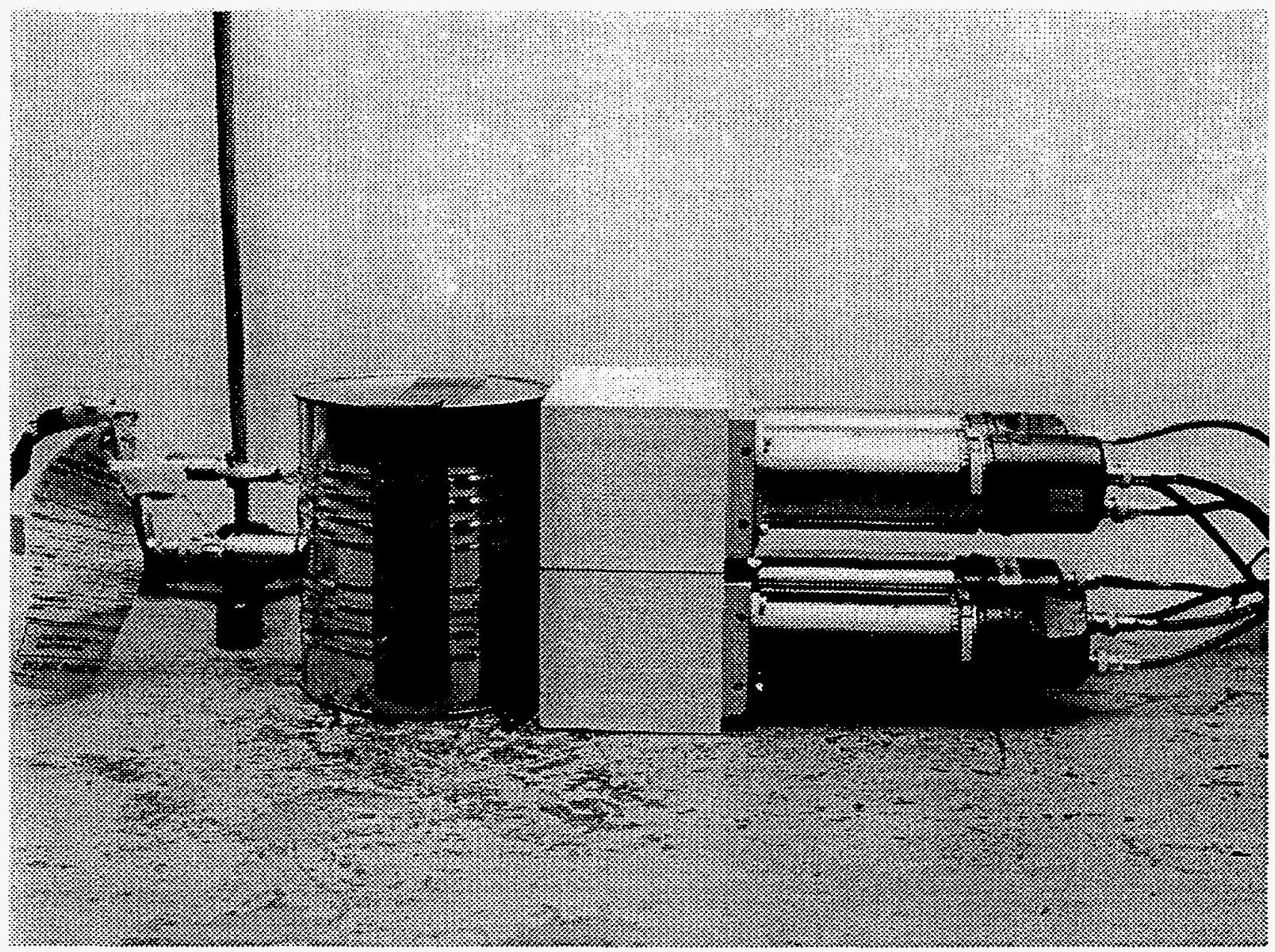

Fig. 1.4. Source-Detector (4)-Casting Configuration for Measurements Without Moderator 


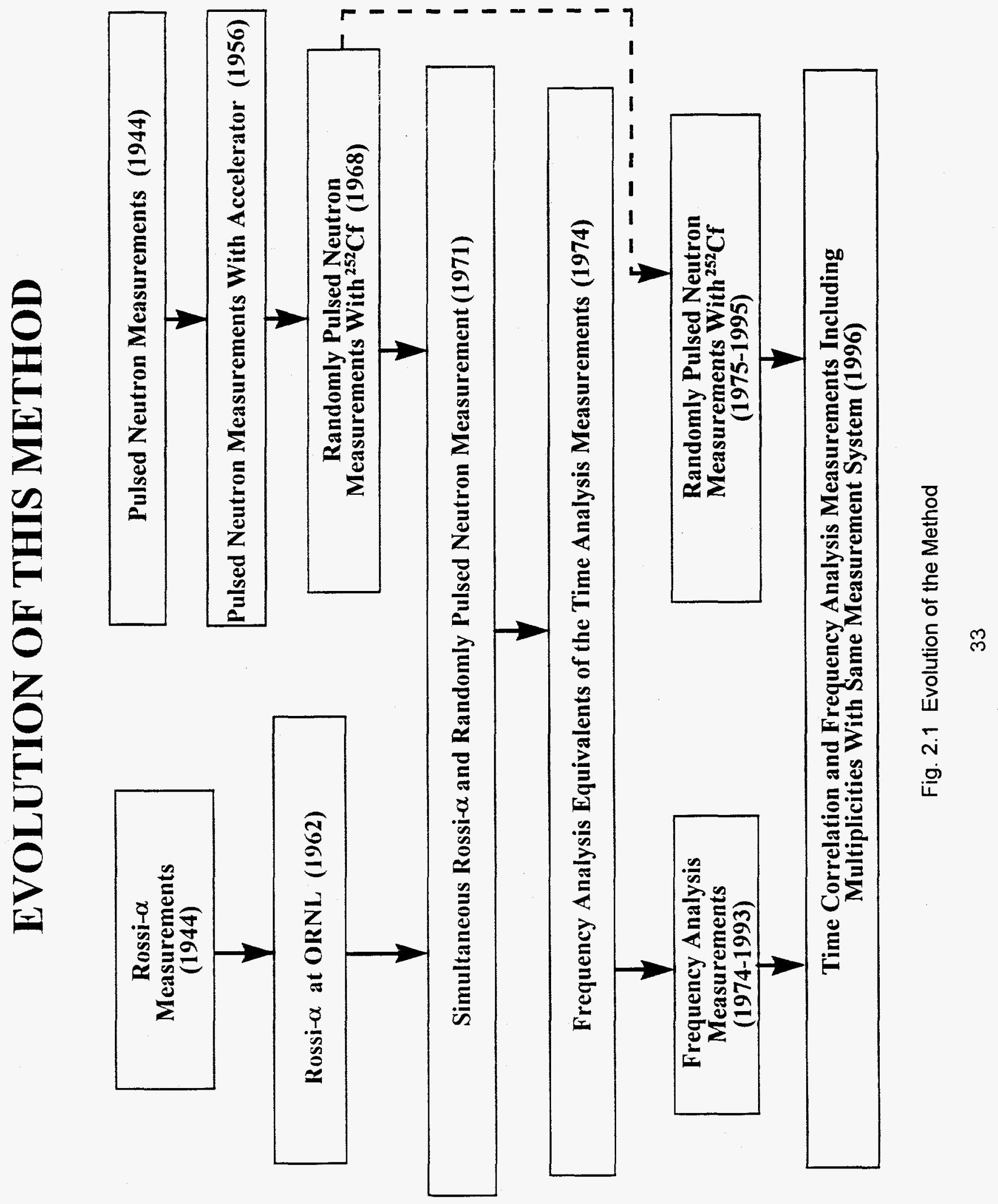




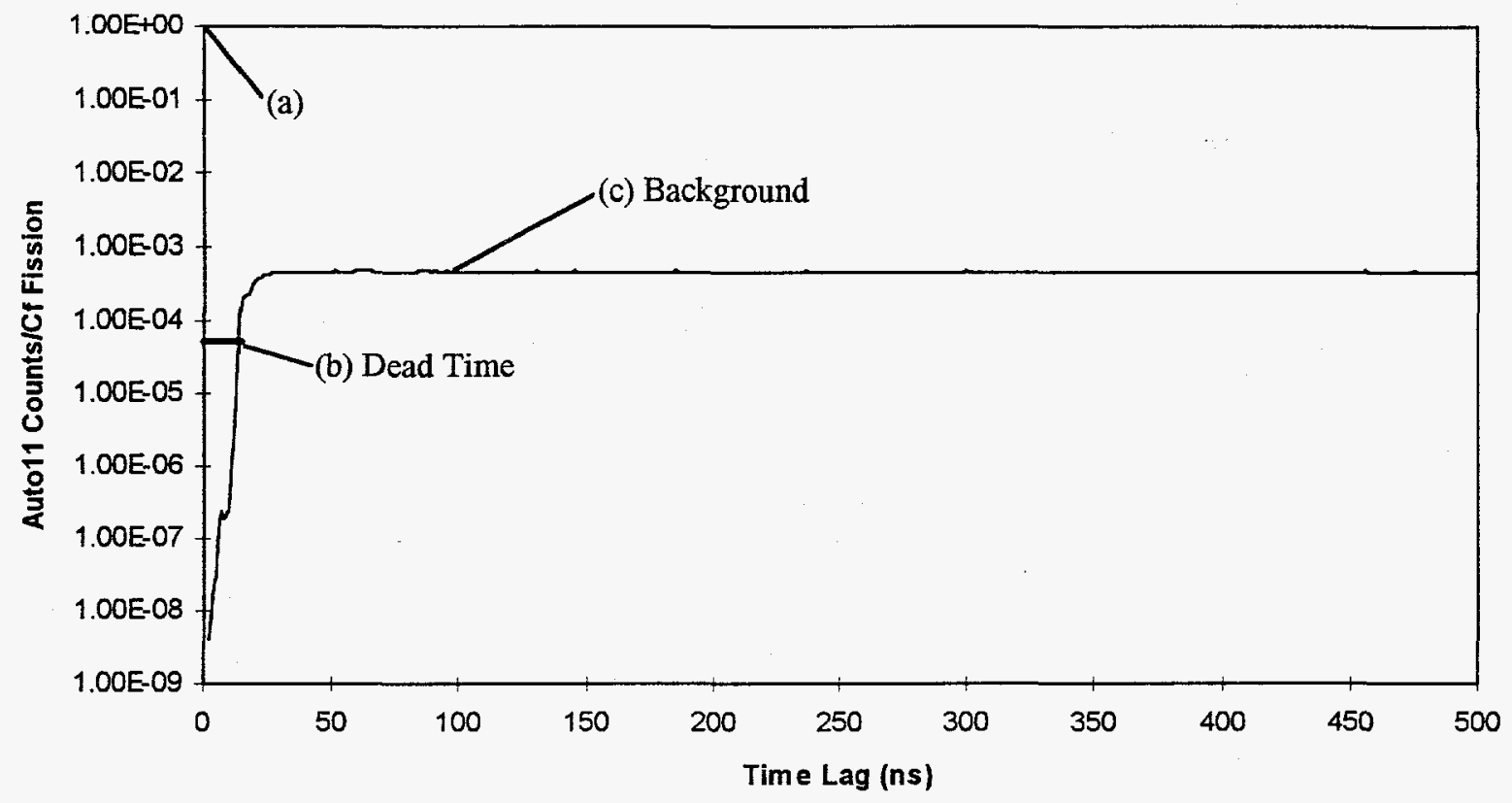

Fig. 5.1. Auto correlation function of the $\mathrm{Cf}$ source ionization chamber detection channel

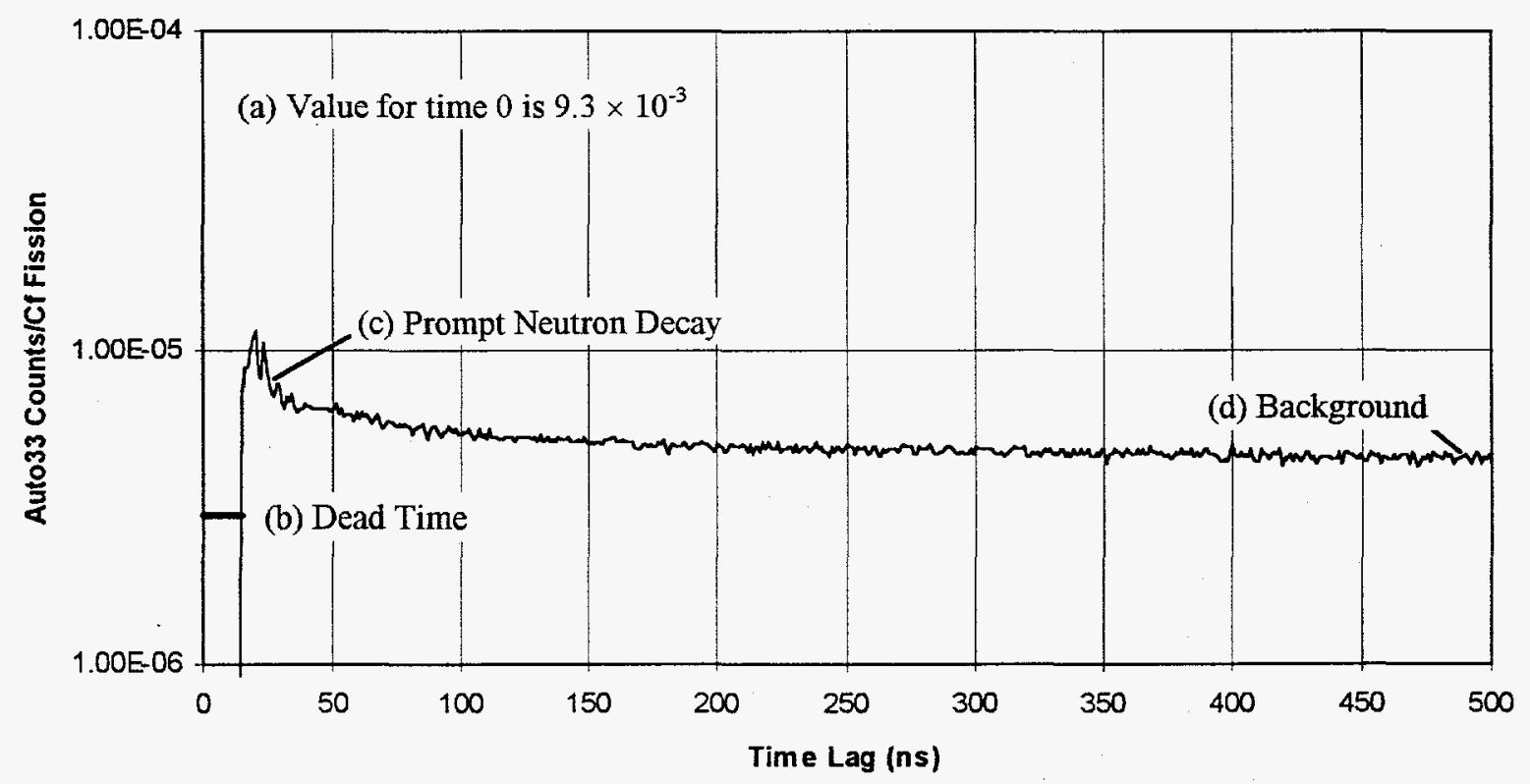

Fig. 6.1. Auto correlation function of a detector channel 


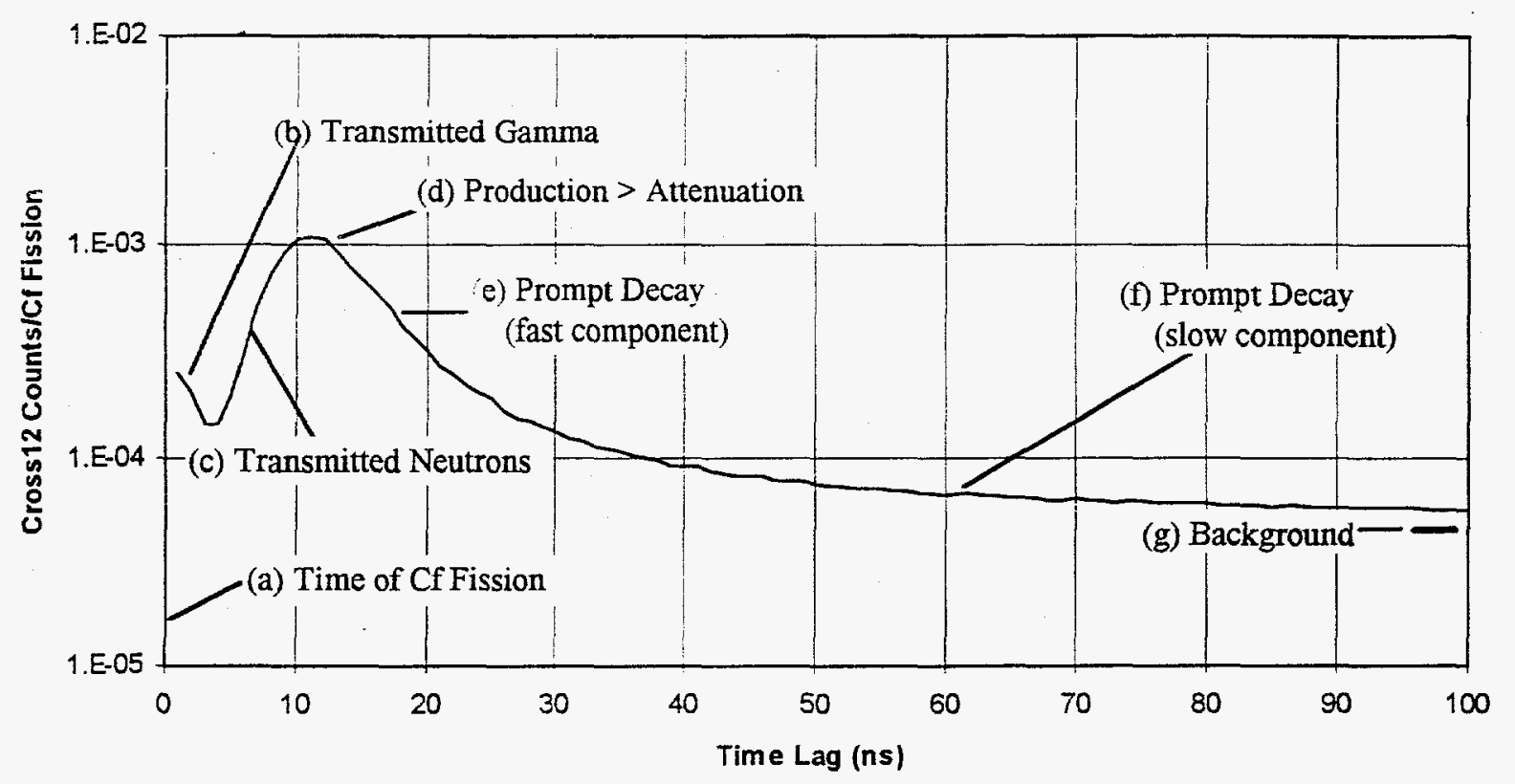

Fig. 7.1. Right half $(\tau \geq 0)$ of the cross correlation function between detector and the Cf source ionization chamber

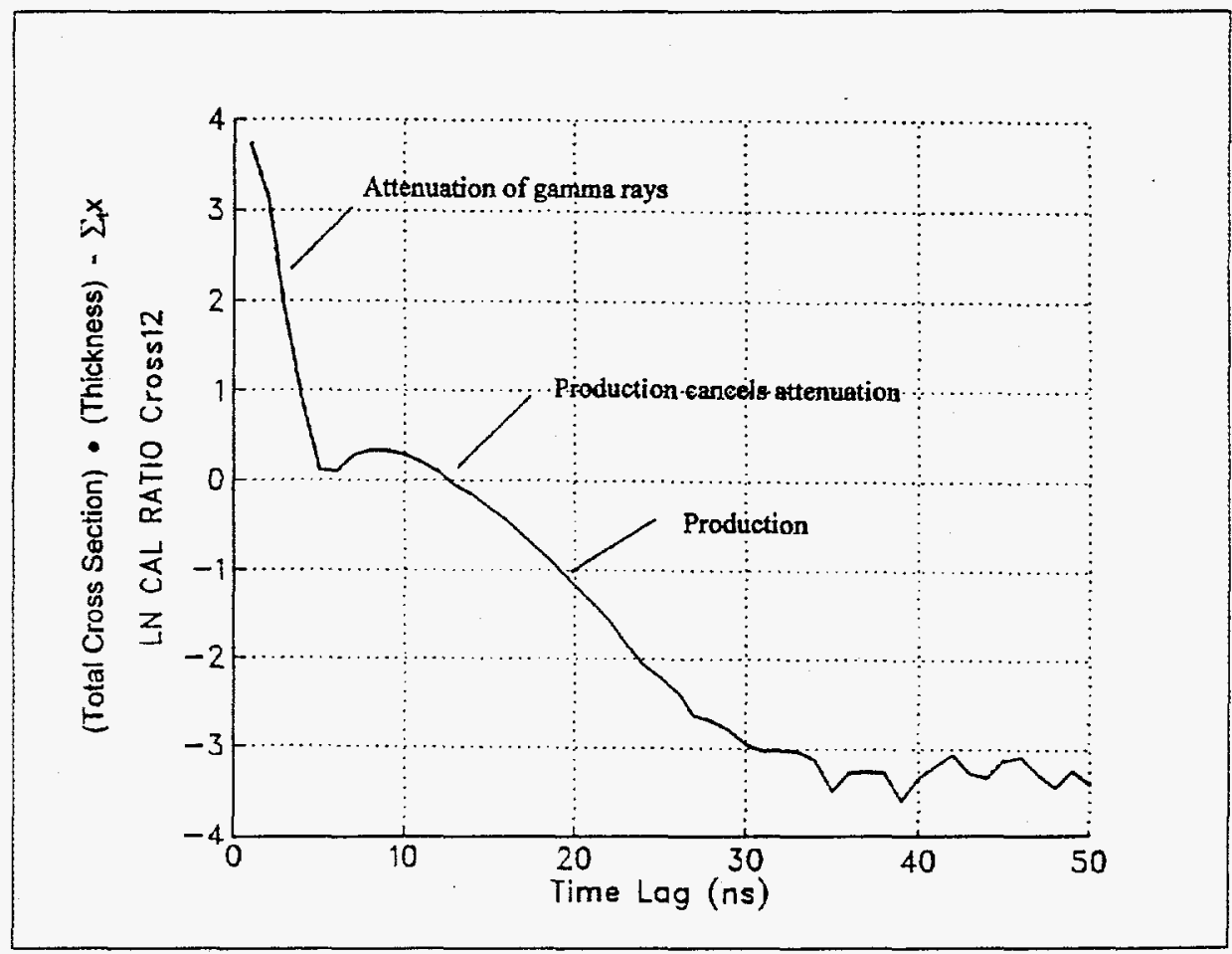

Fig. 7.2. Approximation to $\sum x$ : Logarithm of ratio of cross correlation between source and detector with casting present to source-detector cross correlation in air of the same spacing 


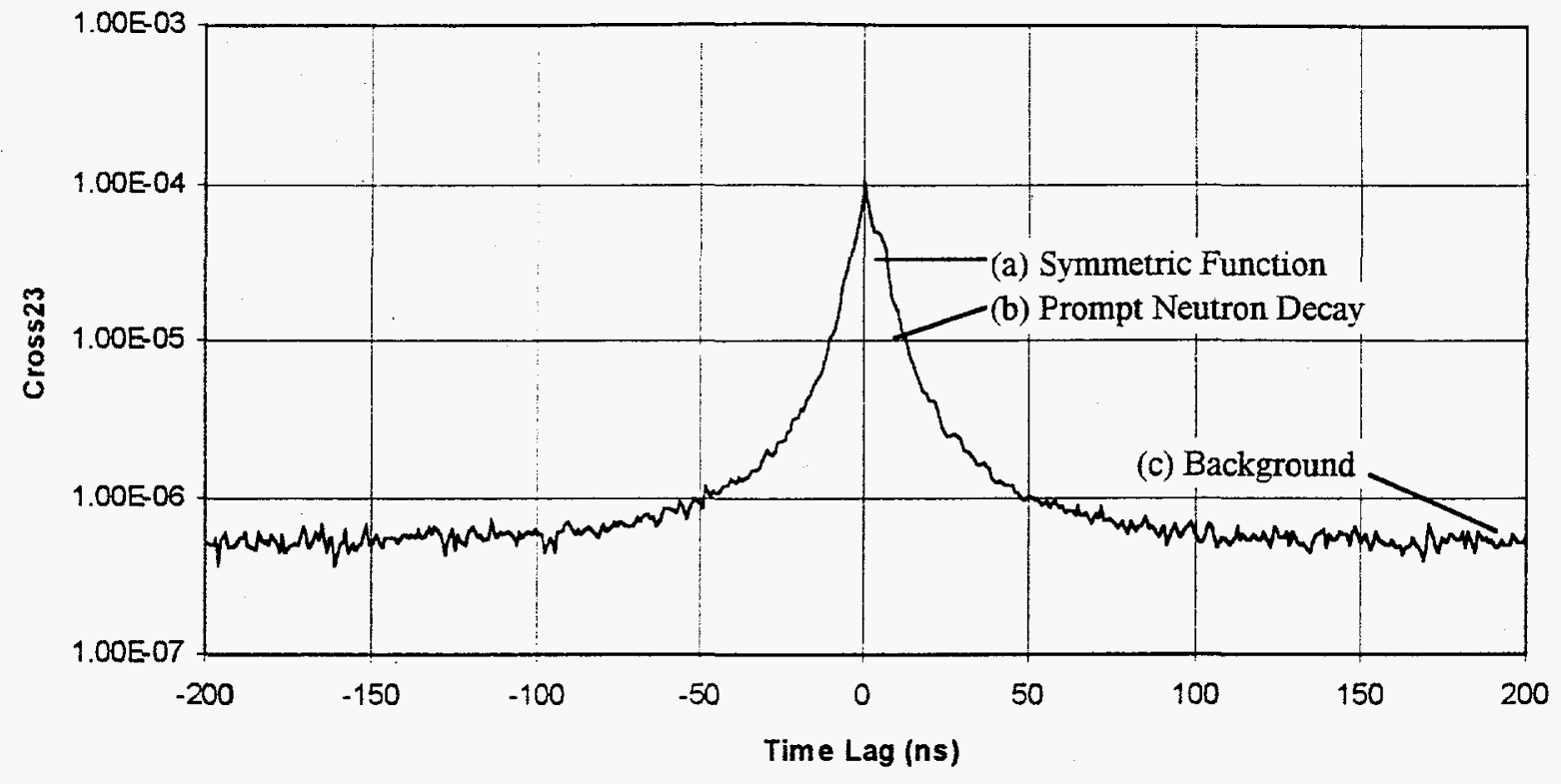

Fig. 8.1. Cross correlation function between two detection channels

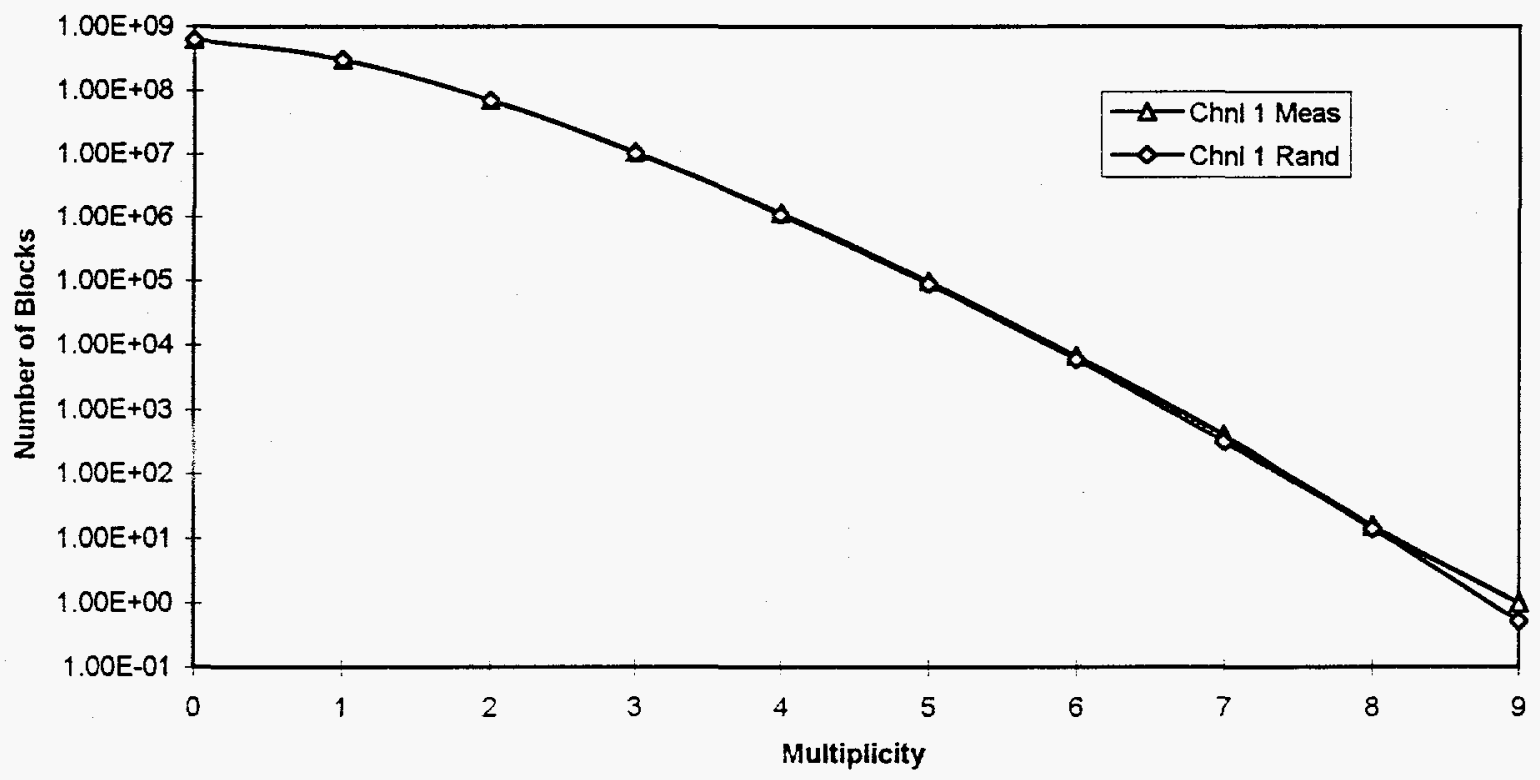

Fig. 9.1. Multiplicities for the source ionization chamber channel 


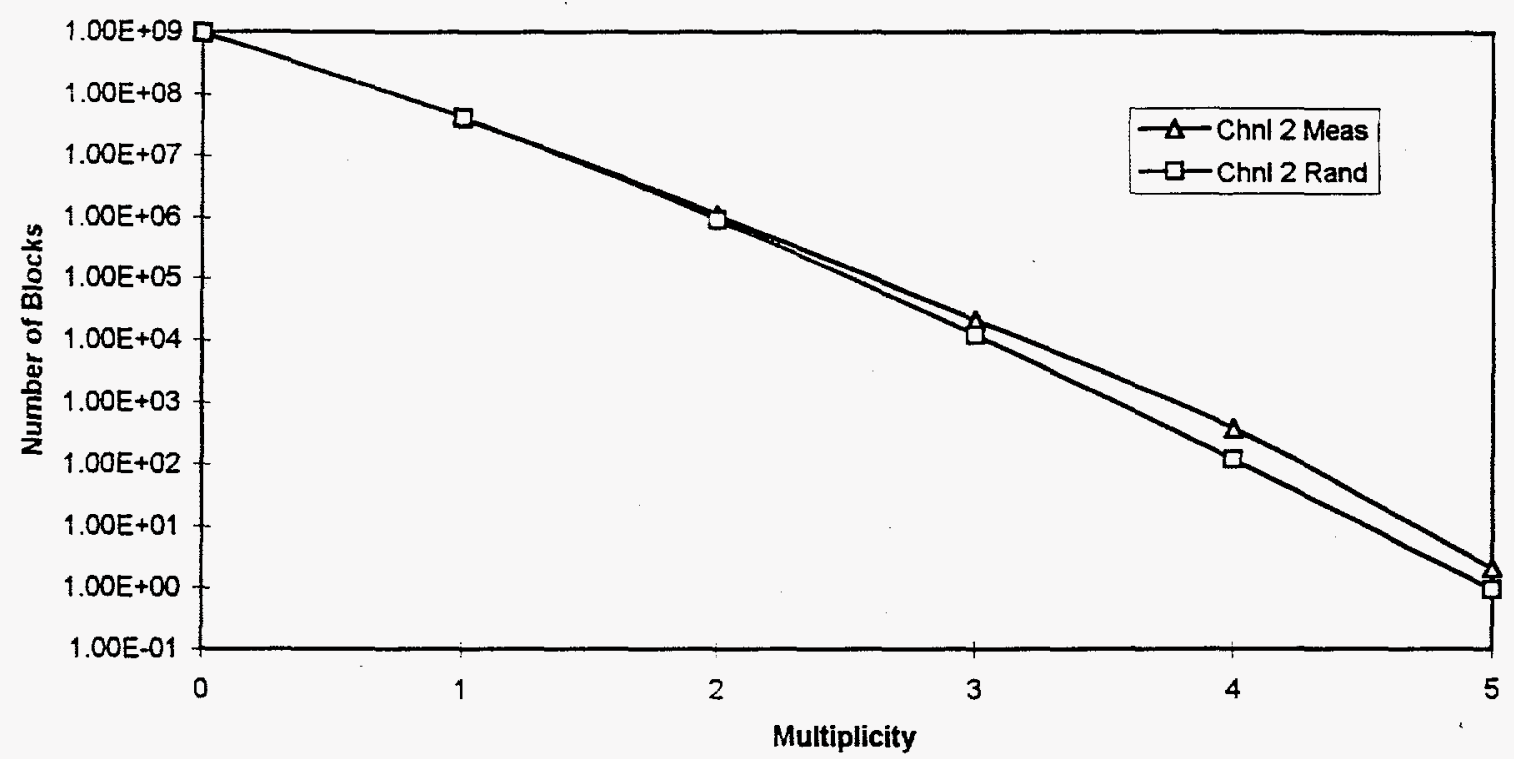

Fig. 9.2. Multiplicities for a single detector channel (2)

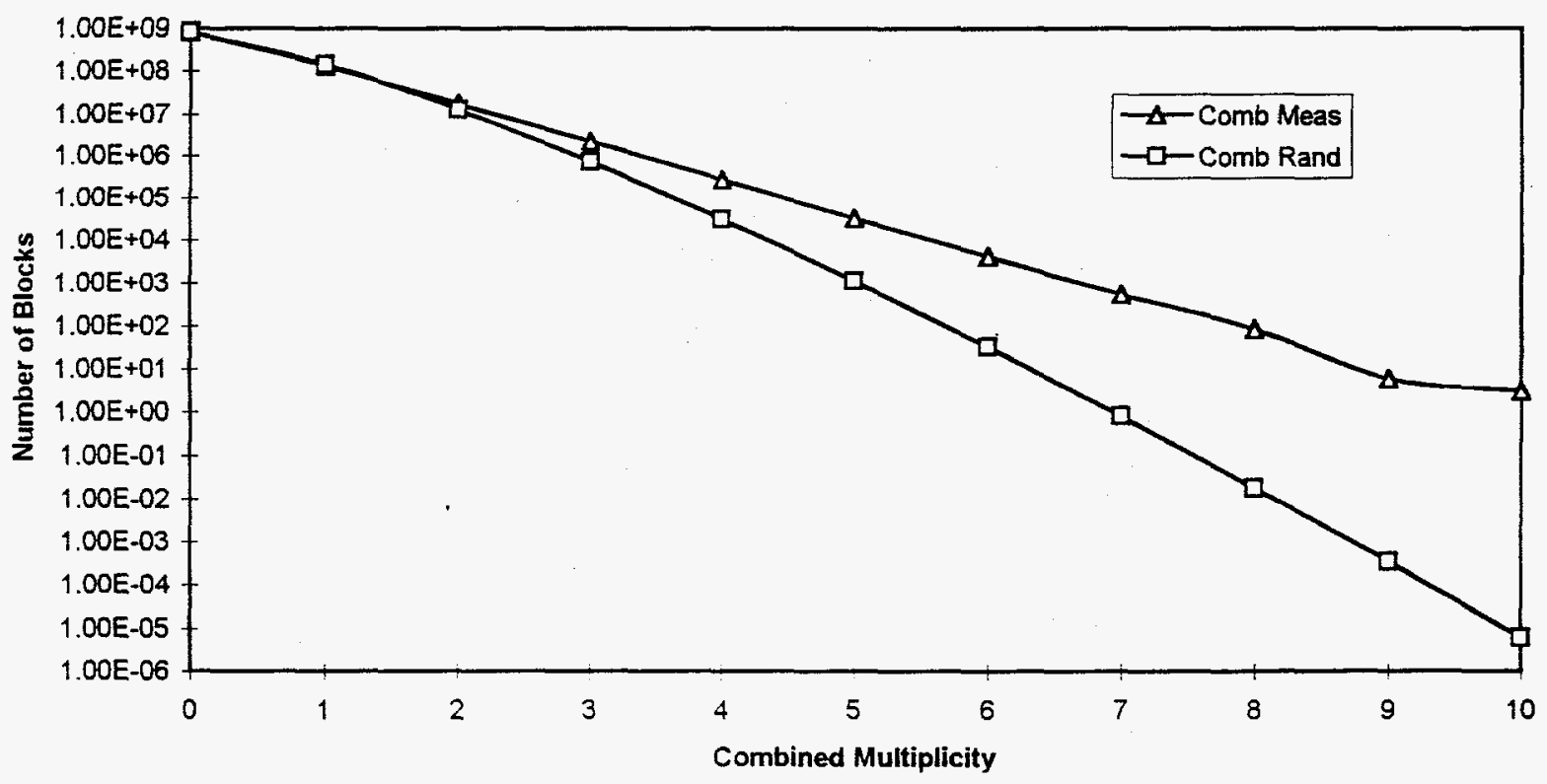

Fig. 9.3. Combined multiplicity for all detector channels 


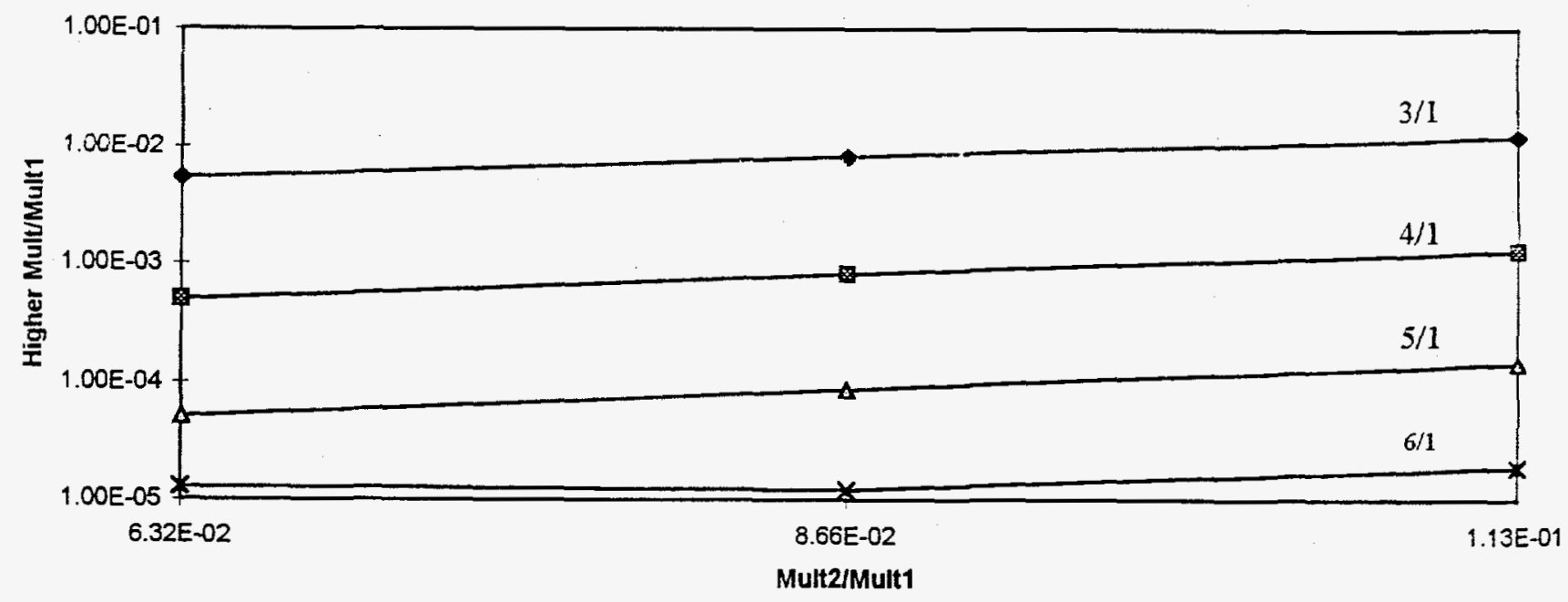

Fig. 9.4. Ratios of higher order/singles as a function of doubles to singles ratio for a uranium casting with varying moderator thickness

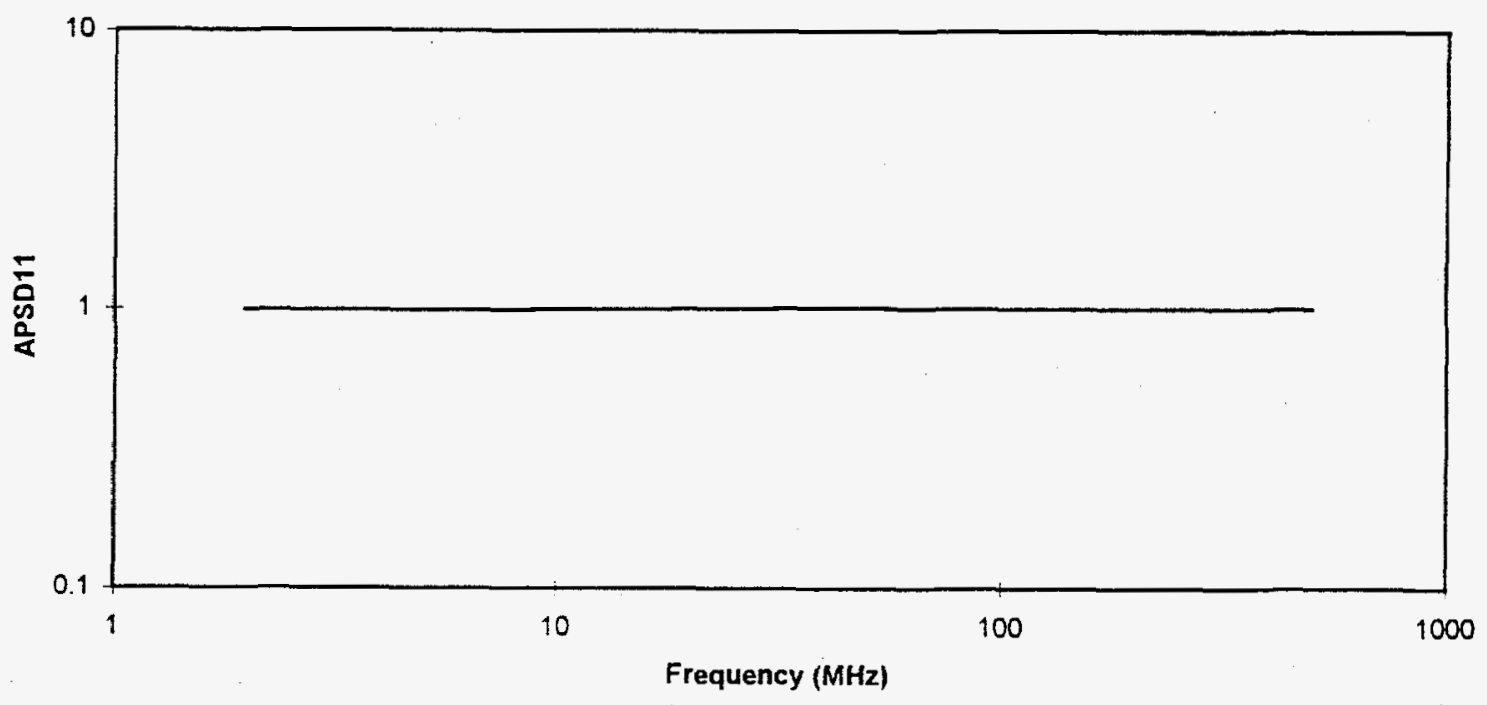

Fig. 10.1. APSD $G_{11}$ of the source detection channel as a function of frequency 


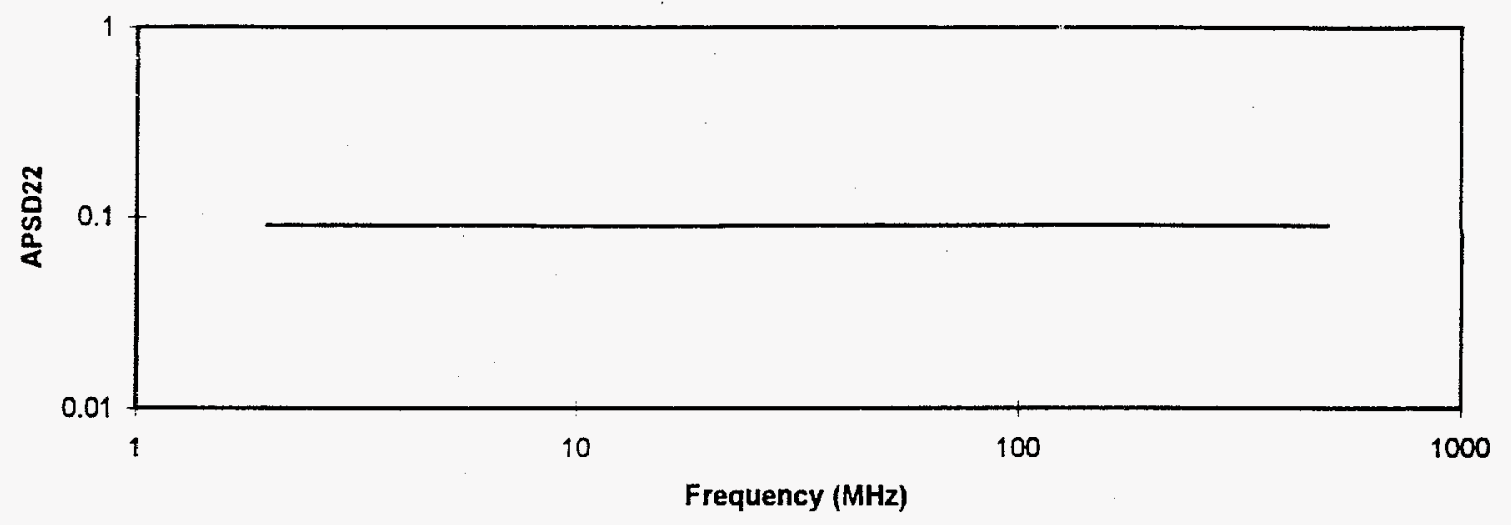

Fig. 10.2. APSD $\mathrm{G}_{22}$ of one of the detector channels

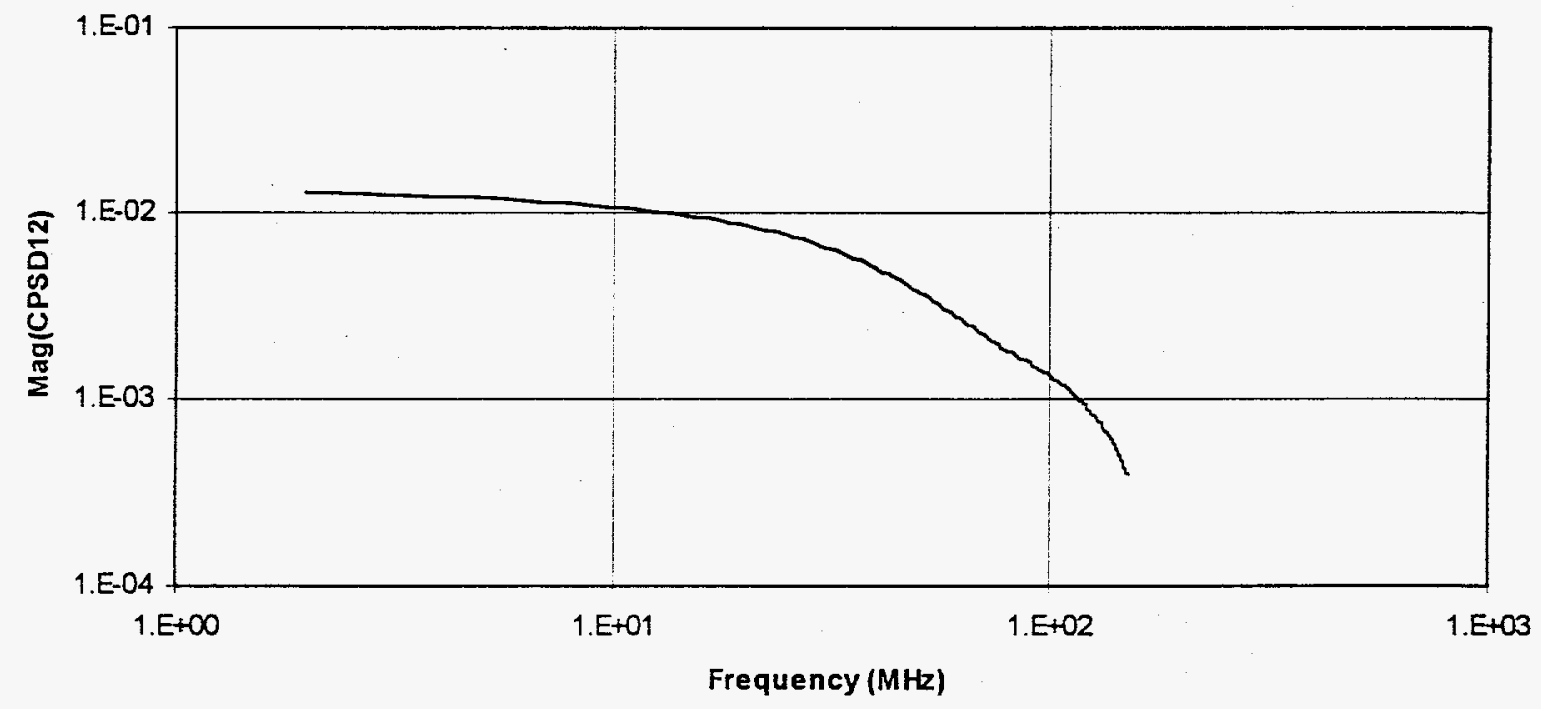

Fig. 11.1. Cross power spectral density between detector channel 2 and the source channel as a function of frequency 


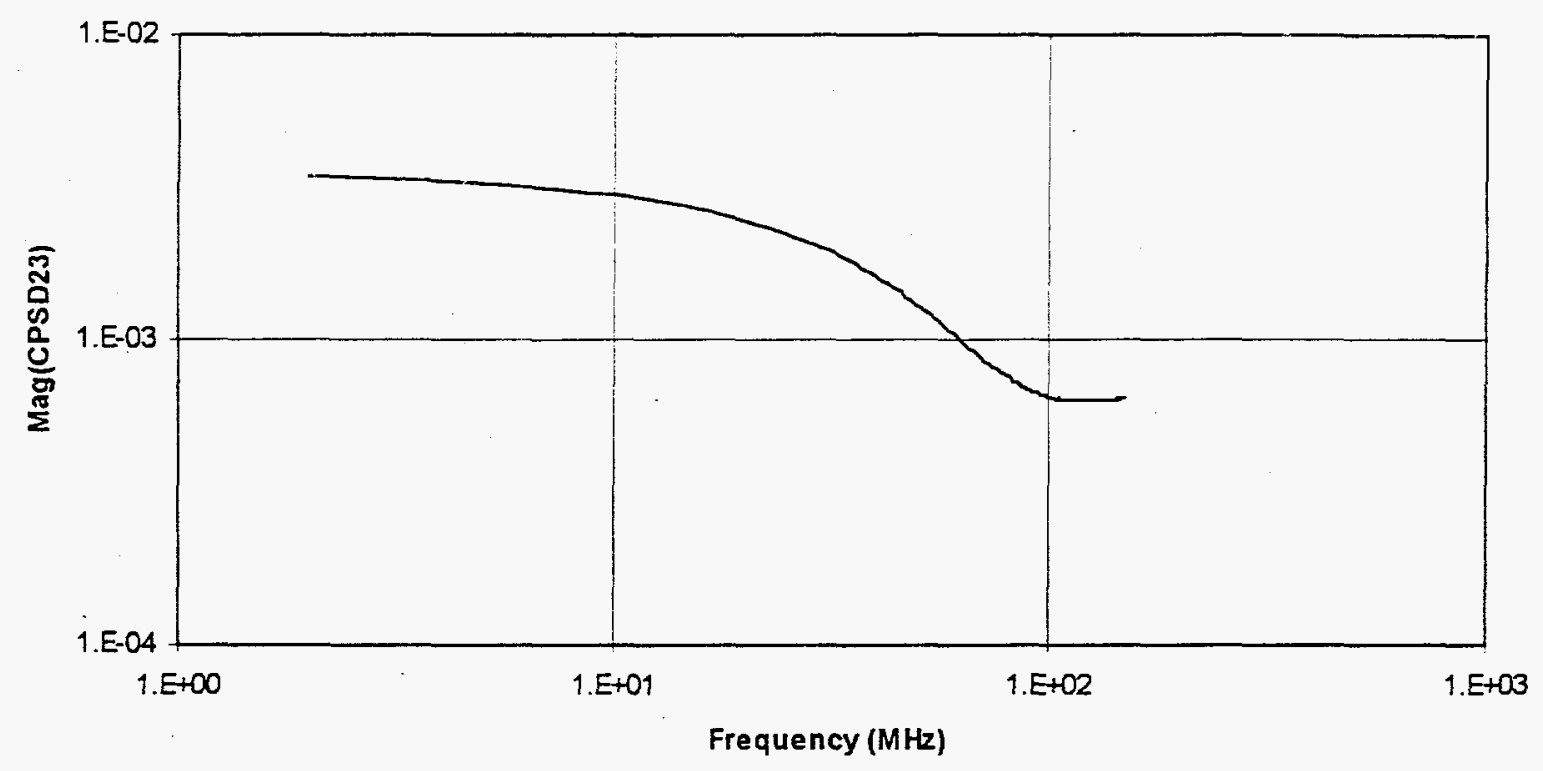

Fig. 11.2. Cross power spectral density between detectors as a function of frequency

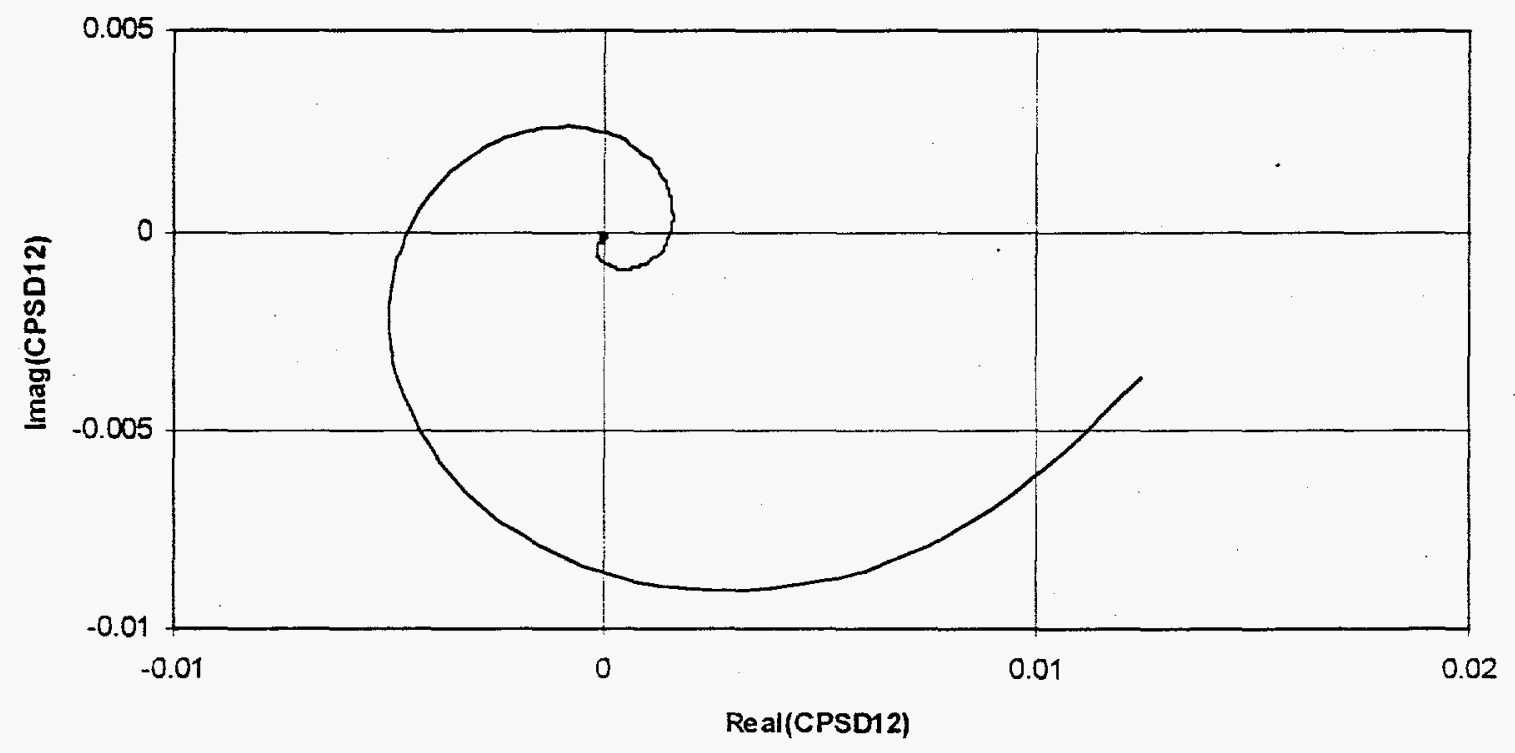

Fig. 11.3. Polar plot of CPSD12 imaginary part vs real part 


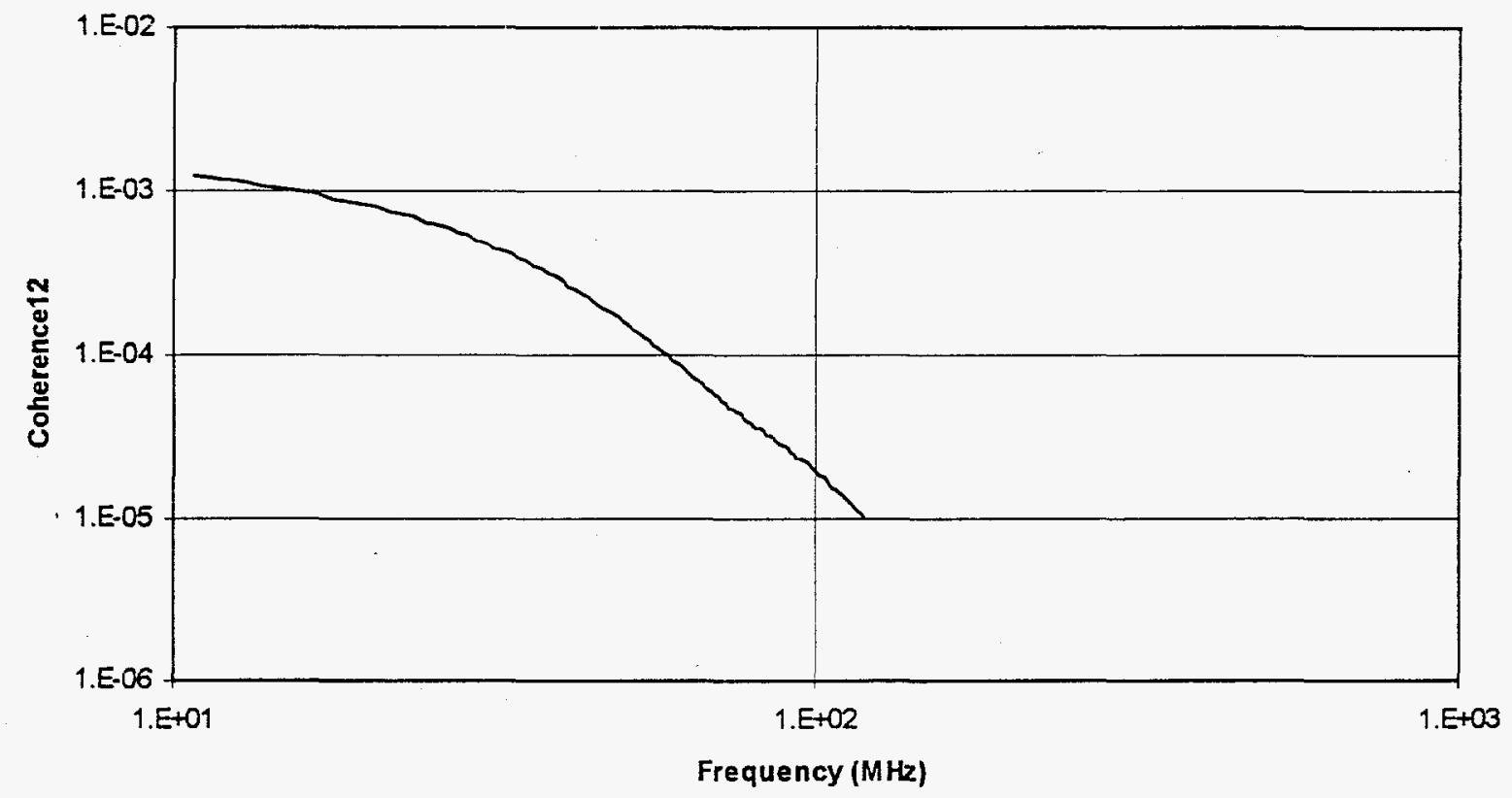

Fig. 12.1. Coherence between a detection channel and the ${ }^{252} \mathrm{Cf}$ source as a function of frequency

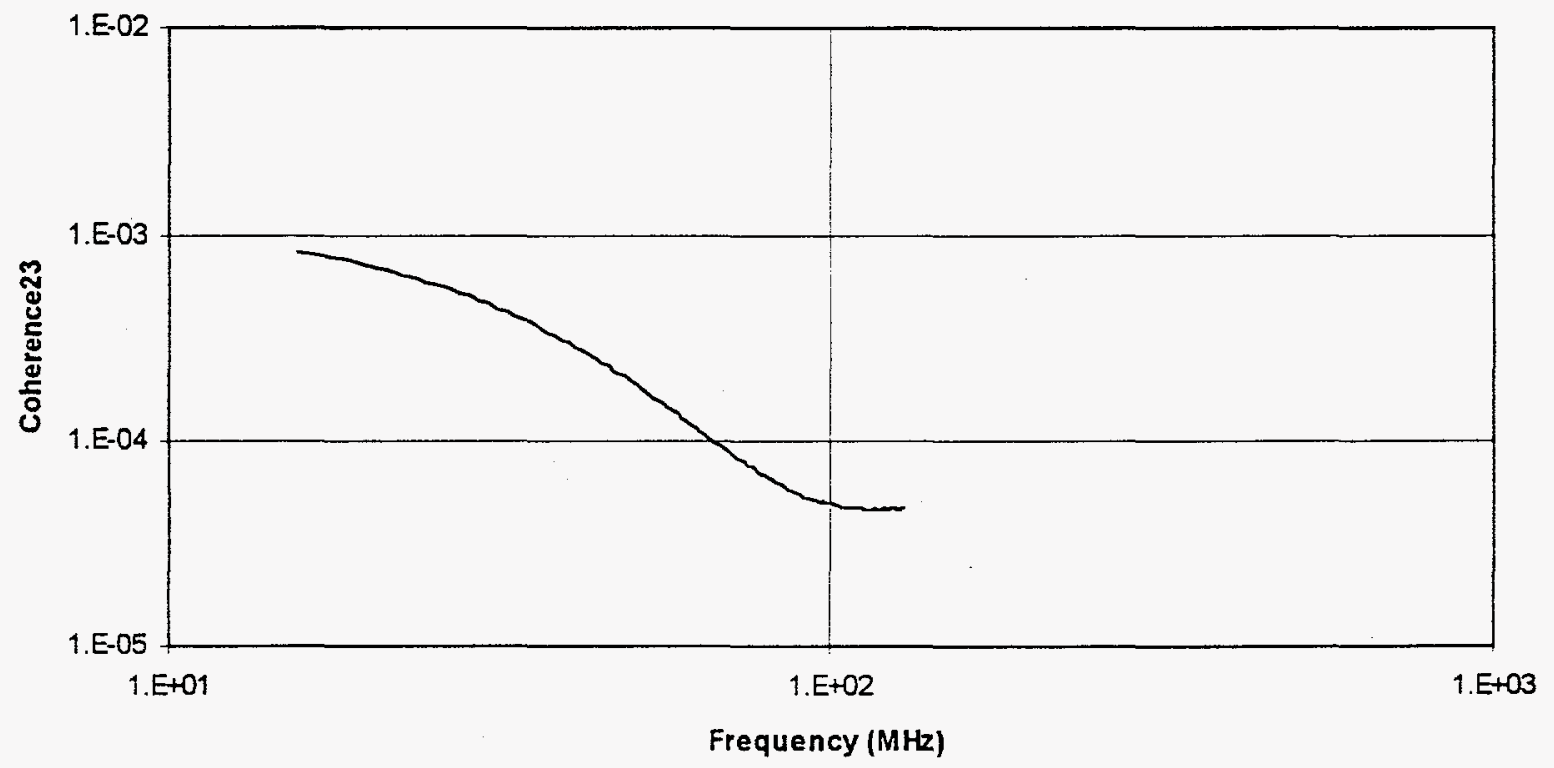

Fig. 12.2. Coherence between the two detection channels as a function of frequency 


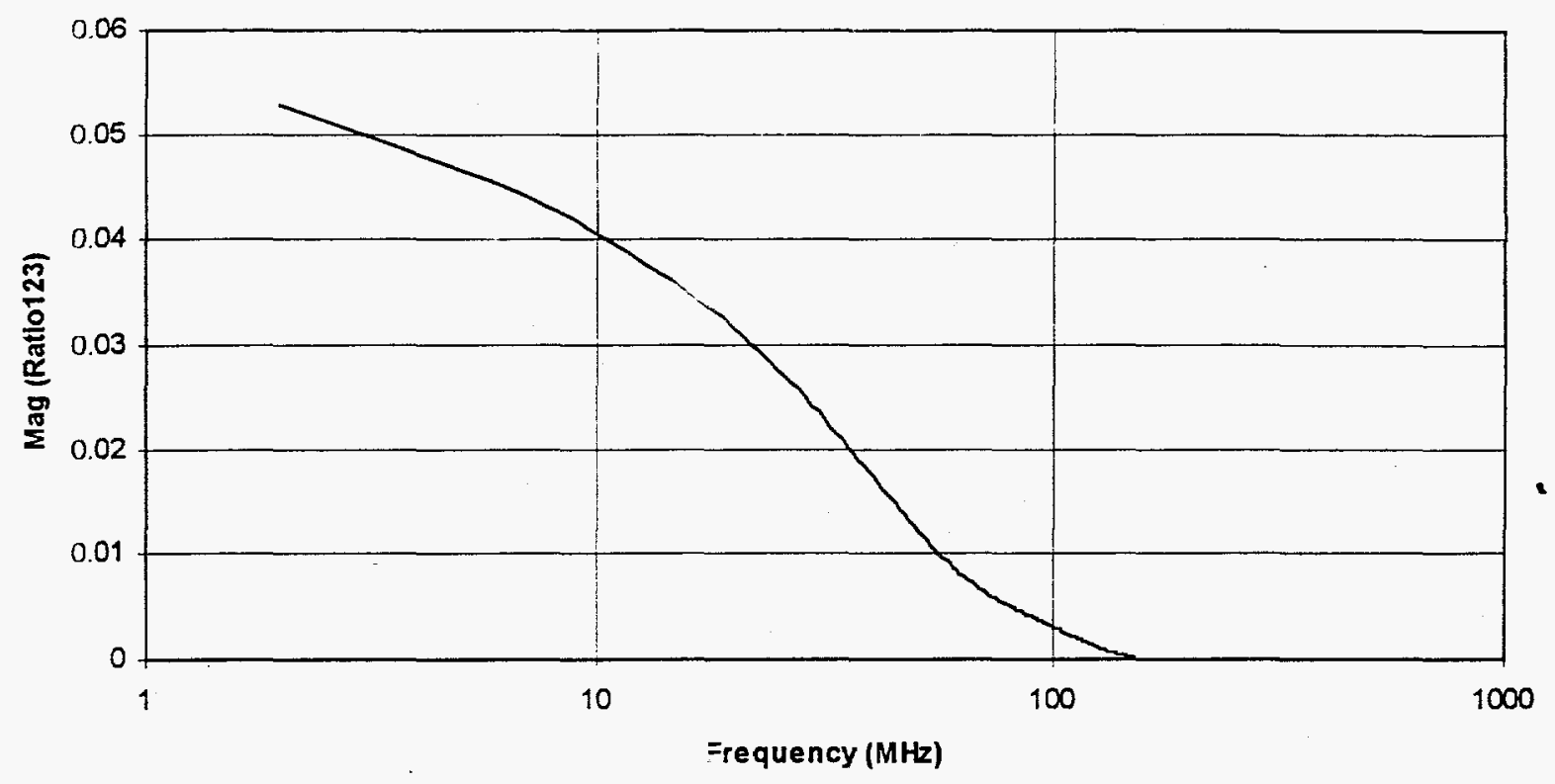

Fig. 13.1. Ratio of spectra densities as a function of frequency

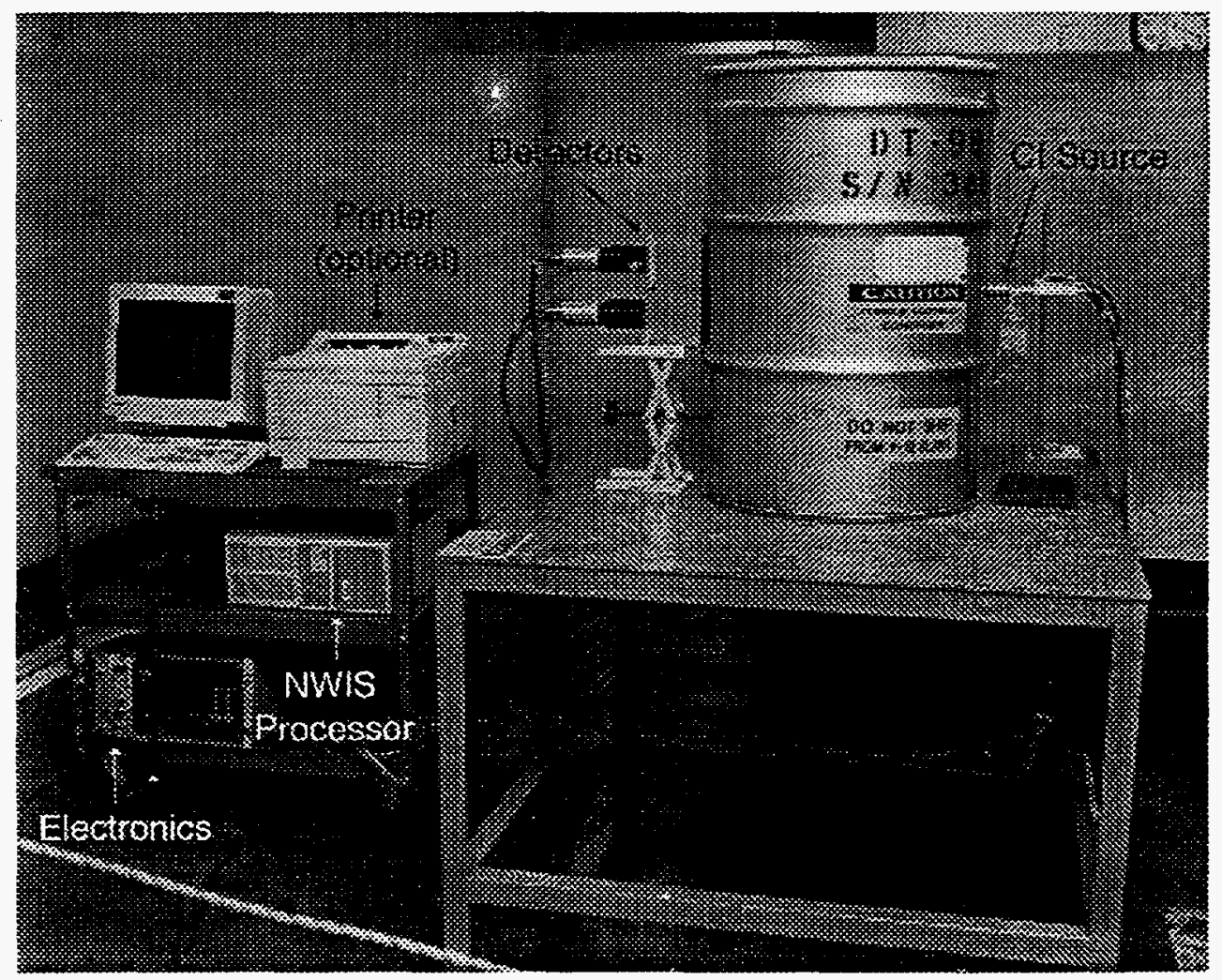

Fis E.1. NWIS Signature Acquisition with Cart Portable System at the Y-12 Plant (1996). 


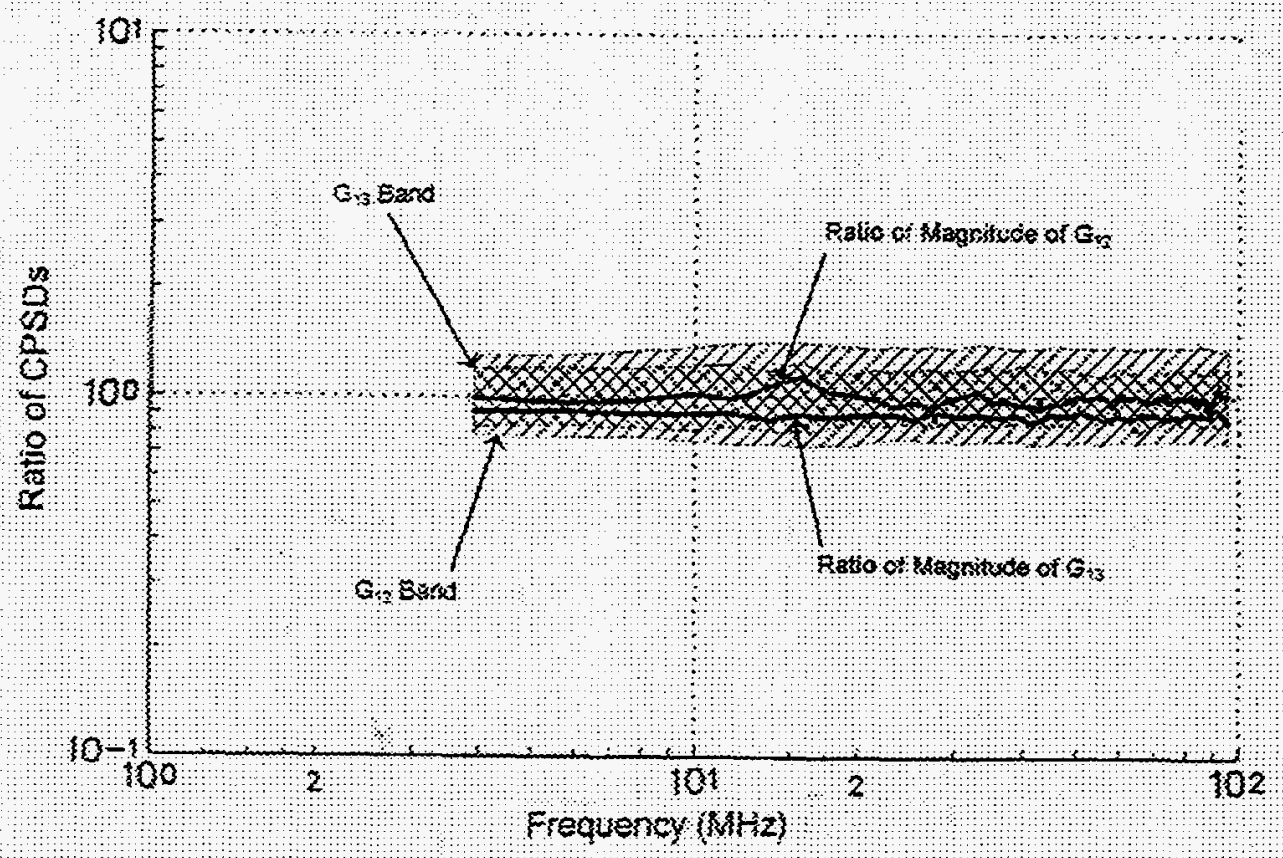

Fig. E.2. Ratios of CPSDs Between Source and Detector to the Reference Set for a Weapons Component in the Set.

(Shaded areas show limits of Reference Set for each of the CPSDs.)

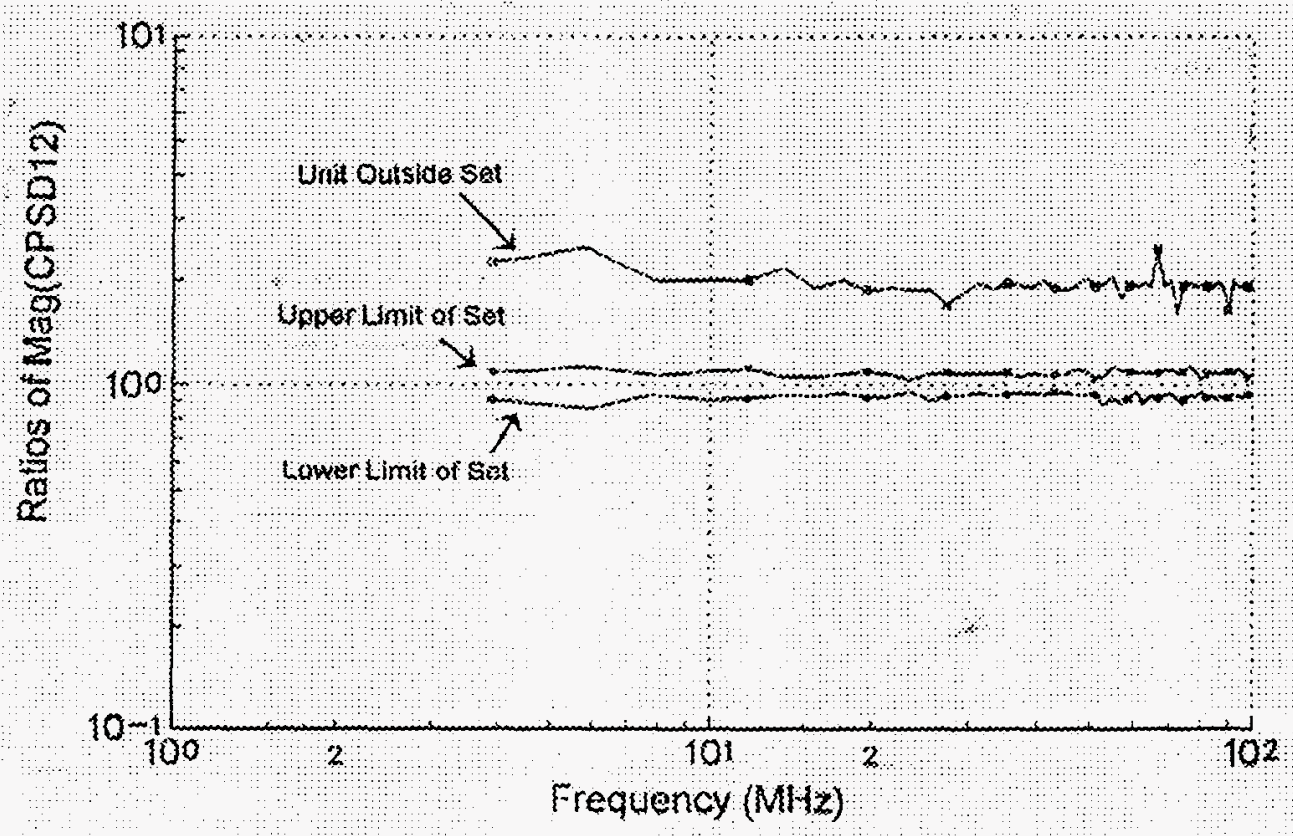

Fig. E.2. Ratio of CPSD Between Source and Detector to the Reference Set for the Weapons Component Not in the Set. 


$$
\text { . }
$$




\section{INTERNAL DISTRIBUTION}

V. M. Baylor

Z. W. Bell

G. N. Cobham

R. N. Ceo

S. A. Cox

J. B. Dooley (10)

M. H. Ehinger

D. N. Fry

T. A. Gafford

R. G. Gilliland

W. H. Hopwood (10)

S. S. Hughes (10)

J. A. March-Leuba

M. J. Maston (5)

J. K. Mattingly

D. W. McDonald
J. A. McEvers

J. T. Mihalzco (30)

J. A. Mullens

R. B. Perez

C. A. Pickett

A. W. Riedy (5)

J. P. Reiner

P. Singh

D. A. Tollefson

T. Uckan

R. E. Uhrig

T. E. Valentine (10)

M. Westfall

J. D. White

R. T. Wood 


\section{EXTERNAL DISTRIBUTION}

A. J. Bieniawski, Negotiations and Analysis Division, Office of Arms Control and Nonproliferation, NN-42, GA-007 (FORSTL), Washington, DC 20585
A. L. Boni,

E. Brag,

L. Bratcher,

A. J. Caffrey,

R. N. Cherry,

B. Crain,

D. W. Crawford,

N. Diaz,

C. E. Dickerman,

D. H. Dye,

D. R. Finch,

L. R. Foulke,

J. L. Fuller,

D. M. Gordon,

T. B. Gosnell,

P. Hezeltine,

J. D. Immele,

J. A. Ingram,

J. D. Jackson,

M. W. Johnson,

J. Kapsales,

M. J. Katz,

South Carolina 29801

Central Intelligence Agency, Washington, D.C. 20505

Pantex, P.O. Box 30020, Amarillo, Texas 79177

Idaho National Engineering Laboratory, Lockheed Martin Idaho

Technologies, P.O. Box 1625, Idaho Falls, Idaho 83415-2114

U.S. DOE, International Safeguards Division, NN-44, GA-045, Forrestal

Building, Washington, DC 20585

Savannah River Operations, Savannah River Site, P.O. Box A,

Aiken, South Carolina 29801

U.S. DOE, 1000 Independence Avenue, S.W., Germantown, NN-512

Washington, DC 20585

Nuclear Regulatory Commission, One White Flint North, 11555 Rockville

Pike, Rockville, MD 20852-2738

Argonne National Laboratory, 9700 South Case Avenue,

Argonne, Illinois 60439

Lawrence Livermore National Laboratory, 7000 East Avenue

Livermore, CA 94550

Savannah River Laboratory, P.O. Box A, Bldg. D-101, Room 735-A, Aiken, South Carolina 29801

Westinghouse Electric Corp., Bettis Atomic Power Laboratory, P. O. Box 79, West Mifflin, PA 15122-0079

Pacific Northwest National Laboratory, 902 Battelle Blvd., P.O. Box 999, Richland, Washington, 99352

Brookhaven National Laboratory, P.O. Box 5000, Upton, NY 11973

Lawrence Livermore National Laboratory, 7000 East Avenue L-175, (366), Livermore, CA 94550

Field Command Defense Special Weapons Agency/FCICV, 160 Texas

Street, SE, Kirtland, New Mexico 87117

U.S. Department of Energy, Headquarters, Forrestal, NN-1, 1000

Independence Avenue, S.W., Washington, D.C. 20585

PANTEX, P.O. Box 30020, Amarillo, TX 79177

U.S. Department of Energy, Oak Ridge Operations Office, Acting Y-12 Site Manager, Bldg. 9704-2, MS-8009

Los Alamos National Laboratory, NIS-6, MS J562, TA-18 Bldg 0129,

Room 12, Los Alamos, NM 87545

U.S. Department of Energy, Headquarters, Germantown, DP-23, 19901 Germantown Road, Germantown, MD 20874-1290

U.S. Department of Energy, Headquarters, Forrestal, DP-15, GA-087, 1000

Independence Avenue, S.W., Washington, D.C. 20585 
F. A. Kloverstrom,

G. F. Knoll,

J. A. Larrimore, Col. G. Lunsford,

R. E. Malenfant,

T. P. McLaughlin,

J. F. Morgan,

M. J. Newman,

N. J. Nichols,

M. F. O'Connell,

B. A. Pohl,

J. C. Pratt,

D. K. Reitzel,

W. L. Riffle,

T. Shea,

G. D. Smith,

D. P. Spears,

J. M. Taylor,

R. Warner,

D. R. Waymire,

P. White,
Lawrence Livermore National Laboratory, 7000 East Avenue Livermore, CA 94550

The University of Michigan, 2355 Bonisteel Blvd. (Cooley), Ann Arbor, MI 48109-2104

International Atomic Energy Agency, P.O. Box 100, Vienna, A-1400 Austria U.S. Department of State, PM/PCI 7320, 2201 C Street NW, Washington, D.C. 20520

U.S. DOE Headquarters, NN-21, GA-293, Forrestal, 1000 Independence Avenue, S. W., Washington, DC 20585

Los Alamos National Laboratory, ESH-5, MS-F691, TA-03 Bldg. 0332, Room 104, Los Alamos, NM 87545

Lawrence Livermore National Laboratory, 7000 East Avenue, L-175

Livermore, CA 94550

U. S. Department of Energy, Headquarters, Forrestal, NN-41, 1000 Independence Avenue, S.W., Washington, D.C. 20585

Los Alamos National Laboratory, NIS-6, MS J562, Los Alamos, NM 87545

U.S. DOE. Forrestal Building, NN-20, MS-7A-049, 1000 Independence

Avenue, S.W., Washington, DC 20585

Lawrence Livermore National Laboratory, 7000 East Avenue, Box 808, MS-L-059 Livermore, CA 94550

Los Alamos National Laboratory, NIS-6, J562, TA-18, Bldg. 0030,

Room 110 Los Alamos, NM 87545

Science Applications International Corporation (SAIC), 2109 Air Park Road S.E., Albuquerque, New Mexico 87106

U.S. DOE Headquarters, Germantown, NN-521, 19901 Germantown Road, Germantown, MD 20874-1290

International Atomic Energy Agency, Wagramerstrasse 5, P.O. Box 100, A-1400 Vienna (Austria)

U.S. DOE Headquarters, Germantown, NN-513, 19901 Germantown Road, Germantown, MD 20874-1290

U. S. Department of Energy, Headquarters, Forrestal, NN-20,

1000 Independence Avenue, S.W., Washington, D.C. 20585

Sandia National Laboratory, Org. 5031, P.O. Box 5800,

Albuquerque, NM 87185

Pacific Northwest National Laboratory, 902 Battelle Blvd., P.O. Box 999, MS K6-48, Richland, Washington 99352

Sandia National Laboratory, Org. 5031, P.O. Box 5800,

Albuquerque, NM 87185

Los Alamos National Laboratory, NIS-CISA, MS K760, TA-66 Bldg 0101, Room D103, Los Alamos, NM 87545 


\section{Distribution:}

Recipients as designated by the author plus:

Y-12 Plant Records Services (3) $9711-5$, MS-8169

[Includes 2 copies for OSTI and 1 copy for Central Files]

Note: This distribution page should not be included in the copy of your document (abstract, visuals, article, etc.) swomitted to a journal or technical society. 